Keywords durability, SB8, elemental concentrations, XRF, DCP, PCT

Retention: permanent

\title{
ANALYTICAL PLANS SUPPORTING THE SLUDGE BATCH 8 GLASS VARIABILITY STUDY BEING CONDUCTED BY ENERGY SOL UTIONS AND CUA'S VITREOUS STATE LABORATORY
}

T.B. Edwards

D.K. Peeler

November 2012

Applied Computational Engineering and Statistics Savannah River National Laboratory

Aiken, SC 29808

This document was prepared in conjunction with work accomplished under Contract No. DE-AC09-08SR22470 with the U.S. Department of Energy.

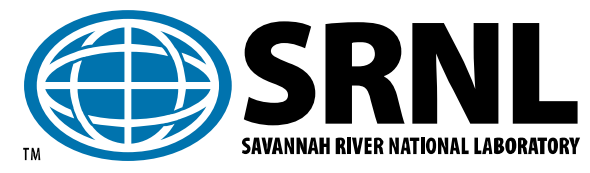


SRNL-STI-2012-00725

Revision 0

DISCLAIMER

This work was prepared under an agreement with and funded by the U.S. Government. Neither the U.S. Government or its employees, nor any of its contractors, subcontractors or their employees, makes any express or implied: 1 . warranty or assumes any legal liability for the accuracy, completeness, or for the use or results of such use of any information, product, or process disclosed; or 2. representation that such use or results of such use would not infringe privately owned rights; or 3. endorsement or recommendation of any specifically identified commercial product, process, or service. Any views and opinions of authors expressed in this work do not necessarily state or reflect those of the United States Government, or its contractors, or subcontractors.

This document was prepared in conjunction with work accomplished under Contract No. DE-AC09-08SR22470 with the U.S. Department of Energy. 


\section{REVIEWS AND APPROVALS}

\section{AUTHORS:}

T. B. Edwards, Applied Computational Engineering and Statistics

Date Savannah River National Laboratory

D. K. Peeler, Process Technology Programs

Date

Savannah River National Laboratory

\section{TECHNICAL REVIEWERS:}

R. A. Baker, Applied Computational Engineering and Statistics

Date

Savannah River National Laboratory

J. W. Amoroso, Process Technology Programs

Date

Savannah River National Laboratory

\section{APPROVERS:}

P. L. Lee, Manager, Applied Computational Engineering and Statistics

Date

Savannah River National Laboratory

C. C. Herman, Manager, Process Technology Programs

Date

Savannah River National Laboratory

E. J. Freed, Manager, Waste Solidification Engineering

Date

Savannah River Remediation 


\section{EXECUTIVE SUMMARY}

EnergySolutions (ES) and its partner, the Vitreous State Laboratory (VSL) of The Catholic University of America (CUA), are to provide engineering and technical services support to Savannah River Remediation, LLC (SRR) for ongoing operation of the Defense Waste Processing Facility (DWPF) flowsheet as well as for modifications to improve overall plant performance. SRR has requested via a statement of work that ES/VSL conduct a glass variability study (VS) for Sludge Batch 8. SRR issued a technical task request (TTR) asking that the Savannah River National Laboratory (SRNL) provide planning and data reduction support for the ES/VSL effort. This document provides two analytical plans for use by ES/VSL: one plan is to guide the measurement of the chemical composition of the study glasses while the second is to guide the measurement of the durability of the study glasses. The measurements generated by ES/VSL are to be provided to SRNL for data reduction and evaluation. SRNL is to review the results of its evaluation with ES/VSL and SRR. The results will subsequently be incorporated into a joint report with ES/VSL as a deliverable to SRR to support the processing of SB8 at DWPF. 


\section{TABLE OF CONTENTS}

LIST OF ABBREVIATIONS ....................................................................................... vi

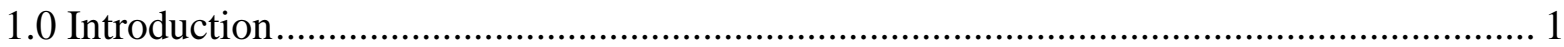

2.0 Analytical Plan for the Measurement of Glass Compositions ......................................... 1

2.1 Naming Conventions for Study Glasses and Prepared Samples .................................. 2

2.2 Sample Preparation for Chemical Composition Analyses ........................................... 3

2.3 Measurement of Chemical Compositions................................................................. 4

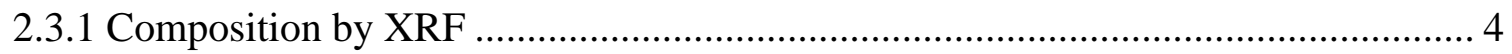

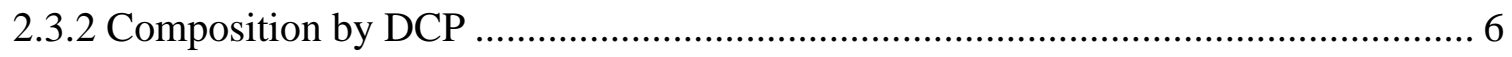

3.0 Analytical Plan for the Measurement of PCT Solutions.................................................. 7

3.1 Grouping of Glasses for Oven Runs................................................................... 7

3.2 Measurement of the PCT Solutions....................................................................... 9

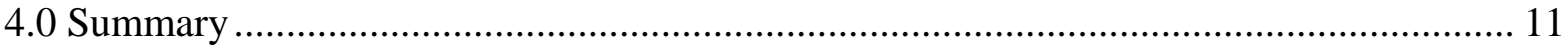

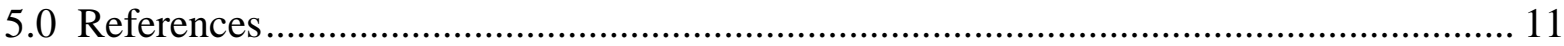

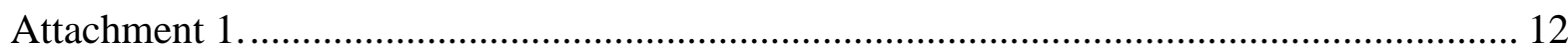

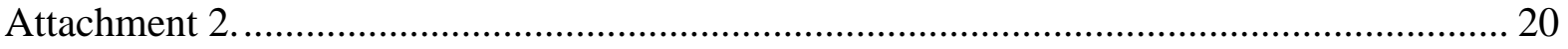




\section{LIST OF ABBREVIATIONS}

$\begin{array}{ll}\text { ANL-LRM } & \text { Argonne National Laboratory - Low Activity Reference Material } \\ \text { ARM-1 } & \text { Approved Reference Material - One (glass) } \\ \text { CUA } & \text { The Catholic University of America } \\ \text { DCP } & \text { Directly Coupled Plasma - Atomic Emission Spectroscopy } \\ \text { DWPF } & \text { Defense Waste Processing Facility } \\ \text { EA } & \text { Environmental Assessment (glass) } \\ \text { ES } & \text { EnergySolutions } \\ \text { HLW } & \text { High-Level Waste } \\ \text { ICP } & \text { Inductively Coupled Plasma - Atomic Emission Spectroscopy } \\ \text { LAW } & \text { Low-Activity Waste } \\ \text { NIST } & \text { National Institute of Standards and Technology } \\ \text { pPm } & \text { parts per million } \\ \text { PCT } & \text { Product Consistency Test } \\ \text { SB8 } & \text { Sludge Batch 8 } \\ \text { SOW } & \text { Statement of Work } \\ \text { SRNL } & \text { Savannah River National Laboratory } \\ \text { SRR } & \text { Savannah River Remediation, LLC } \\ \text { TTR } & \text { Technical Task Request } \\ \text { TTQAP } & \text { Task Technical and Quality Assurance Plan } \\ \text { VS } & \text { Variability Study } \\ \text { VSL } & \text { Vitreous State Laboratory } \\ \text { WTP } & \text { Waste Treatment Plant } \\ \text { XRF } & \text { X-ray Fluorescence Spectroscopy } \\ & \end{array}$




\subsection{INTRODUCTION}

EnergySolutions (ES) and its partner, the Vitreous State Laboratory (VSL) of The Catholic University of America (CUA), are to provide engineering and technical services support to Savannah River Remediation, LLC (SRR) for ongoing operation of the Defense Waste Processing Facility (DWPF) flowsheet as well as for modifications to improve overall plant performance. SRR has requested via a statement of work (SOW) that ES/VSL conduct a glass variability study (VS) for Sludge Batch 8 (SB8) [1]. Specifically, the SOW scope covers several activities including:

- ES/VSL is to batch and fabricate 22 glasses to frame the activities described in this report ${ }^{f}$.

- VSL is to measure the chemical compositions of these study glasses in accordance with an analytical plan, which is to be provided by the Savannah River National Laboratory (SRNL).

- ES/VSL is to conduct two heat treatments for each of the study glasses - a DWPF centerline canister cooled heat treatment and a quenched version.

- ES/VSL is to subject samples of both heat treatments of each study glass to the ASTM Product Consistency Test (PCT) Method A [2] for durability testing.

- The solutions resulting from this testing are to be subjected to chemical analyses by VSL in accordance with an analytical plan, which is to be provided by SRNL.

As seen by the description of these activities, SRNL is to work with ES/VSL to assist in the completion of SRR's SOW. SRNL's support for the SB8 VS was formally requested by SRR in a Technical Task Request (TTR) [3]. As outlined in the Task Technical and Quality Assurance Plan (TTQAP) prepared by SRNL to support the completion of this request [4], two analytical plans are required to assist ES/VSL as they conduct the SB8 VS: one plan is to guide the VSL in the measurement of the chemical composition of the study glasses while the second is to guide the VSL in the measurement of the durability of the study glasses based upon their PCT responses. The purpose of this technical report is to provide these two analytical plans. Section 2 provides the description and background for the plan supporting the measurement of the chemical compositions of the study glasses. Attachment 1 repeats the portions of this chemical composition plan that may be supplied to the laboratory technicians and other appropriate staff to help in the completion of these measurements. Section 3 provides the description and background for the plan supporting the measurement of the solutions resulting from the PCT evaluations of the study glasses. Attachment 2 repeats the portions of the PCT plan that may be supplied to the laboratory technicians and other appropriate staff to help in the completion of the measurement of the PCTs. The measurements generated by VSL are to be provided to SRNL for data reduction and evaluation. SRNL is to reduce these data and review the results with ES/VSL and SRR. The results will be incorporated into a joint report with ES/VSL as a deliverable to SRR to support the processing of SB8 at DWPF.

\subsection{Analytical Plan for the Measurement of Glass Compositions}

The analytical plan needed by ES/VSL to support the measurement of the chemical compositions of the VS glasses is outlined in the sub-sections that follow. Twenty-two (22) glasses containing thorium oxide and depleted uranium oxide are to be identified by SRNL in a separate report for this ES/VSL

\footnotetext{
${ }^{f}$ Discussions with SRR and ES/VSL led to the determination that 22 glasses would be utilized to support the SB8 VS. The target compositions of these 22 glasses are to be provided with the SRNL glass IDs as used here in a separate report (SRNLL3100-2012-00195) that is currently being prepared and that will be issued independently of this report.
} 
study. Table 1 provides a naming convention linking the identifiers that were used by SRNL for these glasses to identifiers that are to be used by ES/VSL as these glasses are batched and fabricated.

Table 1. VSL Identifiers (IDs) for the Study Glasses Linked to the SRNL Glass IDs

\begin{tabular}{||c|c||c|c||}
\hline SRNL ID & ES/VSL ID & SRNL ID & ES/VSL ID \\
\hline \hline SB8VS-01 & VSL-SB8-01 & SB8VS-13 & VSL-SB8-13 \\
\hline SB8VS-02 & VSL-SB8-02 & SB8VS-14 & VSL-SB8-14 \\
\hline SB8VS-03 & VSL-SB8-03 & SB8VS-15 & VSL-SB8-15 \\
\hline SB8VS-04 & VSL-SB8-04 & SB8VS-16 & VSL-SB8-16 \\
\hline SB8VS-05 & VSL-SB8-05 & SB8VS-17 & VSL-SB8-17 \\
\hline SB8VS-06 & VSL-SB8-06 & SB8VS-18 & VSL-SB8-18 \\
\hline SB8VS-07 & VSL-SB8-07 & SB8VS-19 & VSL-SB8-19 \\
\hline SB8VS-08 & VSL-SB8-08 & SB8VS-20 & VSL-SB8-20 \\
\hline SB8VS-09 & VSL-SB8-09 & SB8VS-21 & VSL-SB8-21 \\
\hline SB8VS-10 & VSL-SB8-10 & SB8VS-22 & VSL-SB8-22 \\
\hline SB8VS-11 & VSL-SB8-11 & & \\
\hline SB8VS-12 & VSL-SB8-12 & & \\
\hline \hline
\end{tabular}

\subsection{Naming Conventions for Study Glasses and Prepared Samples}

To provide a level of separation between the targeted compositions of the study glasses and the measurements of the study glasses during the analytical process, the naming convention provided in Table 2 is to be used to establish the identifiers for the samples that are to be submitted for laboratory analysis.

Table 2. VSL Identifiers (IDs) for the Study Glasses Linked to the Sample IDs

\begin{tabular}{|c|c||c|c||}
\hline \hline Glass ID & Sample ID & Glass ID & Sample ID \\
\hline VSL-SB8-01 & SB8-20 & VSL-SB8-13 & SB8-14 \\
\hline VSL-SB8-02 & SB8-16 & VSL-SB8-14 & SB8-13 \\
\hline VSL-SB8-03 & SB8-04 & VSL-SB8-15 & SB8-06 \\
\hline VSL-SB8-04 & SB8-02 & VSL-SB8-16 & SB8-10 \\
\hline VSL-SB8-05 & SB8-12 & VSL-SB8-17 & SB8-17 \\
\hline VSL-SB8-06 & SB8-22 & VSL-SB8-18 & SB8-19 \\
\hline VSL-SB8-07 & SB8-05 & VSL-SB8-19 & SB8-09 \\
\hline VSL-SB8-08 & SB8-18 & VSL-SB8-20 & SB8-01 \\
\hline VSL-SB8-09 & SB8-03 & VSL-SB8-21 & SB8-11 \\
\hline VSL-SB8-10 & SB8-15 & VSL-SB8-22 & SB8-08 \\
\hline VSL-SB8-11 & SB8-07 & & \\
\hline VSL-SB8-12 & SB8-21 & & \\
\hline \hline
\end{tabular}

\subsection{Sample Preparation for Chemical Composition Analyses}

Two methods are to be used to conduct the measurement of the chemical compositions of the study glasses: X-ray Fluorescence Spectroscopy (XRF) and Direct Coupled Plasma Atomic Emission 
Spectroscopy (DCP). Each of the study glasses is to be prepared by VSL in duplicate for each of these two measurement methods. The preparation of a single study glass for XRF analysis involves grinding a sample of the glass to less than 200 mesh and sealing two samples of the resultant glass in different vials in sufficient quantities for subsequent XRF analysis. A labeling and ordering scheme for these vials that are to be submitted for XRF is provided in Table 3 . The groupings and sequencing of these glasses that are indicated in this table also guide the glass fabrication process in that the glasses must be batched and melted before the activities of Table 3 may be pursued. Thus, the SB8 VS glasses are to be batched and fabricated in the order as indicated by Table 3.

Table 3. XRF Preparation Blocks and Sequencing along with Sample Identifiers

\begin{tabular}{||l|l||l|l||l|l||}
\hline \hline \multicolumn{2}{||l||}{ XRF Preparation Block 1 } & \multicolumn{2}{|c|}{ XRF Preparation Block 2 } & XRF Preparation Block 3 \\
\hline SB8-05xrf-1 & SB8-05xrf-2 & SB8-16xrf-1 & SB8-16xrf-2 & SB8-02xrf-1 & SB8-02xrf-2 \\
\hline SB8-18xrf-1 & SB8-18xrf-2 & SB8-04xrf-1 & SB8-04xrf-2 & SB8-12xrf-1 & SB8-12xrf-2 \\
\hline SB8-03xrf-1 & SB8-03xrf-2 & SB8-22xrf-1 & SB8-22xrf-2 & SB8-15xrf-1 & SB8-15xrf-2 \\
\hline SB8-21xrf-1 & SB8-21xrf-2 & SB8-10xrf-1 & SB8-10xrf-2 & SB8-07xrf-1 & SB8-07xrf-2 \\
\hline SB8-06xrf-1 & SB8-06xrf-2 & SB8-17xrf-1 & SB8-17xrf-2 & SB8-14xrf-1 & SB8-14xrf-2 \\
\hline SB8-19xrf-1 & SB8-19xrf-2 & SB8-01xrf-1 & SB8-01xrf-2 & SB8-13xrf-1 & SB8-13xrf-2 \\
\hline SB8-09xrf-1 & SB8-09xrf-2 & SB8-11xrf-1 & SB8-11xrf-2 & SB8-20xrf-1 & SB8-20xrf-2 \\
\hline SB8-08xrf-1 & SB8-08xrf-2 & & & & \\
\hline \hline
\end{tabular}

In addition to the grinding and sieving steps used to prepare samples for XRF, the preparation of samples for DCP analysis involves a chemical dissolution step. Once a sample of a study glass has been ground to less than 200 mesh and the two samples of the ground glass have been prepared for XRF analysis, an adequate quantity (to be determined by VSL) of the ground glass is to be placed in a separate vial for subsequent duplicate preparation for analysis by DCP. Duplicate samples from each of the vials are to be subjected to microwave-assisted total acid dissolution in Teflon vessels according to VSL standard operating procedures. Typically, a mixture of concentrated HF: $\mathrm{HNO}_{3}$ is used to conduct the digestions, with each digestion leading to a $50 \mathrm{ml}$ solution. The resulting solutions are further diluted to $200 \mathrm{ml}$ before DCP analysis. The $200 \mathrm{ml}$ solutions are to be labeled as indicated in Table 4. The groupings of Table 4 are established in a manner that indicates that all of the batching and preliminary preparations (i.e., grinding) of the glasses are to be completed before the dissolution activities of Table 4 are initiated. Each block of Table 4 represents a microwave batch, and the sequence provides an order for sample weighing.

In addition to the study glasses, a sample of a standard reference glass (to be selected by VSL) is to be prepared once for repeated analysis by XRF (see Section 2.3.1), and similarly, samples of this standard glass are to be prepared (i.e., dissolved for DCP analysis) to provide sufficient quantities to allow aliquots of the resulting solutions to be submitted for DCP analysis (see Section 2.3.2). It is anticipated that two preparations of the standard glass (Std-dcp) may be needed and these have been included in the preparation blocks of Table 4. If aliquots of the second dissolution of the Std-dcp standard are needed to support the DCP analyses of Section 2.3.2, this should be noted as part of the information provided to SRNL along with the reported measurements. 
SRNL-STI-2012-00725

Revision 0

Table 4. DCP Preparation Blocks and Sequencing along with Sample Identifiers for the $200 \mathrm{ml}$ Solutions

\begin{tabular}{|c|c|}
\hline DCP Preparation Block 1 & DCP Preparation Block 2 \\
\hline SB8-07dcp-1 & SB8-05dcp-1 \\
\hline SB8-13dcp-1 & SB8-05dcp-2 \\
\hline SB8-07dcp-2 & SB8-04dcp-1 \\
\hline SB8-15dcp-1 & SB8-19dcp-1 \\
\hline SB8-16dcp-1 & SB8-04dcp-2 \\
\hline SB8-15dcp-2 & SB8-19dcp-2 \\
\hline SB8-17dcp-1 & SB8-10dcp-1 \\
\hline SB8-13dcp-2 & SB8-22dcp-1 \\
\hline SB8-14dcp-1 & Std-dcp \\
\hline SB8-16dcp-2 & SB8-08dcp-1 \\
\hline SB8-06dcp-1 & SB8-09dcp-1 \\
\hline Std-dcp & SB8-09dcp-2 \\
\hline SB8-02dcp-1 & SB8-18dcp-1 \\
\hline SB8-03dcp-1 & SB8-22dcp-2 \\
\hline SB8-21dcp-1 & SB8-12dcp-1 \\
\hline SB8-01dcp-1 & SB8-08dcp-2 \\
\hline SB8-14dcp-2 & SB8-11dcp-1 \\
\hline SB8-17dcp-2 & SB8-10dcp-2 \\
\hline SB8-03dcp-2 & SB8-12dcp-2 \\
\hline SB8-21dcp-2 & SB8-18dcp-2 \\
\hline SB8-06dcp-2 & SB8-11dcp-2 \\
\hline SB8-20dcp-1 & \\
\hline SB8-02dcp-2 & \\
\hline SB8-20dcp-2 & \\
\hline SB8-01dcp-2 & \\
\hline & \\
\hline
\end{tabular}

\subsection{Measurement of Chemical Compositions}

Once samples of the study glasses and standard glass have been prepared, they are to be submitted for chemical analysis by one of the two methods: XRF or DCP. The resulting measurements are to be provided to SRNL in elemental weight percent. A measurement below its detection limit should be indicated in this table by a less than sign (“<”) followed by the detection limit.

\subsubsection{Composition by XRF}

Measurements for the following elements of interest are to be acquired by VSL using XRF: aluminum $(\mathrm{Al})$, barium $(\mathrm{Ba})$, calcium $(\mathrm{Ca})$, cerium $(\mathrm{Ce})$, chromium $(\mathrm{Cr})$, copper $(\mathrm{Cu})$, iron $(\mathrm{Fe})$, potassium $(\mathrm{K})$, lanthanum (La), magnesium (Mg), manganese (Mn), sodium (Na), nickel (Ni), lead (Pb), sulfur (S), silicon (Si), thorium (Th), titanium (Ti), uranium (U), zinc (Zn), and zirconium (Zr) concentrations. It should be noted that some of the elements listed above are minor components of the study glasses and may be below the detection limits of the analytical procedure. It should also be noted that this is a complete list of elements of interest for this glass study with the exception of boron (B) and lithium (Li); the measurement of these elements is addressed in the next section. 
Blocking and randomizing the glass samples of Table 3 that are to be submitted for XRF measurement are primary concerns in the development of the analytical plan. It is anticipated that the day-to-day variations of the XRF instrumentation may be a minor source of uncertainty for the analytical procedures used to determine the elemental concentrations for the submitted glass samples. However, to provide the data necessary to confirm this, each pair of duplicate samples are to be analyzed by XRF on one day and then remeasured on a subsequent day. A randomized plan that incorporates this approach in measuring the elemental concentrations of the glass samples prepared for XRF analysis is provided in Table 5. An ARL 9400 or other equivalent wavelength dispersive $\mathrm{XRF}$ spectrometer is to be used for this purpose. The spectrometer is to be calibrated over a range of glass compositions using standard reference materials traceable to the National Institute of Standards and Technology (NIST), as well as waste glasses including the Argonne National Laboratory - Low Activity Reference Material (ANL-LRM), the Defense Waste Processing Facility - Environmental Assessment (DWPF-EA) glass, and the Waste Treatment Plant (WTP) High-Level Waste (HLW) and Low-Activity Waste (LAW) glasses. A single sample of a reference glass, that is to be selected by VSL to be a matrix-match for this study and that is to be labeled as "Std-xrf", is to be submitted repeatedly for measurement along with the samples of the study glasses as indicated in Table 5 . The "known" composition of this reference glass is to be provided to SRNL along with the measured compositions from Table 5. These compositions are to be provided to SRNL as weight percent (wt\%) elemental concentrations.

Table 5. XRF Analytical Blocks and Sequencing along with the Measurement Identifiers

\begin{tabular}{|c|c|c|c|c|c|}
\hline $\begin{array}{c}\text { XRF Block 1 } \\
\text { First Set of } \\
\text { Measurements }\end{array}$ & $\begin{array}{c}\text { XRF Block 1 } \\
\text { Second Set of } \\
\text { Measurements }\end{array}$ & $\begin{array}{c}\text { XRF Block 2 } \\
\text { First Set of } \\
\text { Measurements }\end{array}$ & $\begin{array}{c}\text { XRF Block } 2 \\
\text { Second Set of } \\
\text { Measurements }\end{array}$ & $\begin{array}{c}\text { XRF Block } 3 \\
\text { First Set of } \\
\text { Measurements }\end{array}$ & $\begin{array}{c}\text { XRF Block } 3 \\
\text { Second Set of } \\
\text { Measurements }\end{array}$ \\
\hline Std-xrf-111 & Std-xrf-121 & Std-xrf-211 & Std-xrf-221 & Std-xrf-311 & Std-xrf-321 \\
\hline SB8-21xrf-11 & SB8-09xrf-22 & SB8-16xrf-11 & SB8-17xrf-12 & SB8-12xrf-11 & SB8-20xrf-22 \\
\hline SB8-19xrf-11 & SB8-08xrf-12 & SB8-04xrf-11 & SB8-04xrf-12 & SB8-15xrf-21 & SB8-12xrf-12 \\
\hline SB8-08xrf-11 & SB8-05xrf-22 & SB8-11xrf-11 & SB8-11xrf-12 & SB8-12xrf-21 & SB8-20xrf-12 \\
\hline SB8-03xrf-11 & SB8-03xrf-12 & SB8-11xrf-21 & SB8-10xrf-22 & SB8-13xrf-11 & SB8-12xrf-22 \\
\hline SB8-18xrf-11 & SB8-06xrf-12 & SB8-22xrf-21 & SB8-16xrf-12 & SB8-02xrf-21 & SB8-15xrf-12 \\
\hline SB8-06xrf-21 & SB8-06xrf-22 & SB8-10xrf-11 & SB8-01xrf-22 & SB8-13xrf-21 & SB8-07xrf-12 \\
\hline SB8-21xrf-21 & SB8-18xrf-12 & SB8-16xrf-21 & SB8-01xrf-12 & SB8-02xrf-11 & SB8-14xrf-12 \\
\hline SB8-05xrf-21 & SB8-21xrf-12 & SB8-01xrf-21 & SB8-10xrf-12 & SB8-20xrf-11 & SB8-07xrf-22 \\
\hline Std-xrf-112 & Std-xrf-122 & Std-xrf-212 & Std-xrf-222 & Std-xrf-312 & Std-xrf-322 \\
\hline SB8-19xrf-21 & SB8-19xrf-12 & SB8-17xrf-21 & SB8-16xrf-22 & SB8-14xrf-21 & SB8-15xrf-22 \\
\hline SB8-03xrf-21 & SB8-08xrf-22 & SB8-10xrf-21 & SB8-22xrf-22 & SB8-07xrf-21 & SB8-14xrf-22 \\
\hline SB8-18xrf-21 & SB8-19xrf-22 & SB8-04xrf-21 & SB8-22xrf-12 & SB8-20xrf-21 & SB8-13xrf-22 \\
\hline SB8-09xrf-21 & SB8-18xrf-22 & SB8-01xrf-11 & SB8-11xrf-22 & SB8-14xrf-11 & SB8-02xrf-22 \\
\hline SB8-06xrf-11 & SB8-03xrf-22 & SB8-22xrf-11 & SB8-17xrf-22 & SB8-15xrf-11 & SB8-02xrf-12 \\
\hline SB8-09xrf-11 & SB8-05xrf-12 & SB8-17xrf-11 & SB8-04xrf-22 & SB8-07xrf-11 & SB8-13xrf-12 \\
\hline SB8-08xrf-21 & SB8-21xrf-22 & Std-xrf-213 & Std-xrf-223 & Std-xrf-313 & Std-xrf-323 \\
\hline SB8-05xrf-11 & SB8-09xrf-12 & & & & \\
\hline Std-xrf-113 & Std-xrf-123 & & & & \\
\hline
\end{tabular}

The sample identifiers in Table 5 have been modified to provide a labeling scheme for the measurements that are to be generated by these XRF determinations. Specifically, a suffix has been 
added to the label for the sample of the standard glass, Std-xrf, to indicate the XRF block (i.e., 1, 2, or 3), the measurement set (i.e., 1 (first) or 2 (second)), and its position (i.e., 1, 2, or 3) in the set. Finally, a suffix has been added to the sample identifier for the study glasses that indicates the measurement set (i.e., 1 or 2 ).

\subsubsection{Composition by DCP}

The samples prepared for chemical analysis by DCP are to be measured for their boron and lithium content. Each of the duplicate preparations of each study glass is to be measured twice by DCP, with a re-calibration of the instrument being performed between the two measurements. The groupings and sequencing of these samples for DCP analysis are provided in Table 6. To repeat, each column of this table indicates a different calibration of the DCP instrumentation. In addition, the order of the samples in a column is the sequence in which the samples should be measured by DCP. Also, note the addition of aliquots of the prepared samples of the reference glass, Std-dcp, to these DCP analytical blocks. The "known" composition of this reference glass (anticipated to be the same standard as used for the XRF analyses) is to be provided to SRNL along with the measured compositions from Table 6. These compositions are to be provided as weight percent (wt\%) elemental concentrations.

Table 6. DCP Analytical Blocks and Sequencing along with the Measurement Identifiers

\begin{tabular}{|c|c|c|c|c|c|}
\hline $\begin{array}{c}\text { DCP Block 1 } \\
\text { First } \\
\text { Calibration }\end{array}$ & $\begin{array}{c}\text { DCP Block } 1 \\
\text { Second } \\
\text { Calibration }\end{array}$ & $\begin{array}{c}\text { DCP Block } 2 \\
\text { First } \\
\text { Calibration }\end{array}$ & $\begin{array}{c}\text { DCP Block } 2 \\
\text { Second } \\
\text { Calibration }\end{array}$ & $\begin{array}{c}\text { DCP Block } 3 \\
\text { First } \\
\text { Calibration }\end{array}$ & $\begin{array}{c}\text { DCP Block } 3 \\
\text { Second } \\
\text { Calibration }\end{array}$ \\
\hline Std-dcp-111 & Std-dcp-121 & Std-dcp-211 & Std-dcp-221 & Std-dcp-311 & Std-dcp-321 \\
\hline SB8-21dcp-11 & SB8-09dcp-22 & SB8-01dcp-21 & SB8-11dcp-12 & SB8-12dcp-11 & SB8-20dcp-22 \\
\hline SB8-19dcp-11 & SB8-08dcp-12 & SB8-16dcp-11 & SB8-17dcp-12 & SB8-15dcp-21 & SB8-12dcp-12 \\
\hline SB8-08dcp-11 & SB8-05dcp-22 & SB8-04dcp-11 & SB8-04dcp-12 & SB8-12dcp-21 & SB8-20dcp-12 \\
\hline SB8-03dcp-11 & SB8-03dcp-12 & SB8-11dcp-11 & SB8-10dcp-22 & SB8-13dcp-11 & SB8-12dcp-22 \\
\hline SB8-18dcp-11 & SB8-06dcp-12 & SB8-11dcp-21 & SB8-16dcp-12 & SB8-02dcp-21 & SB8-15dcp-12 \\
\hline SB8-06dcp-21 & SB8-06dcp-22 & SB8-22dcp-21 & SB8-01dcp-22 & SB8-13dcp-21 & SB8-07dcp-12 \\
\hline SB8-21dcp-21 & SB8-18dcp-12 & SB8-10dcp-11 & SB8-01dcp-12 & SB8-02dcp-11 & SB8-14dcp-12 \\
\hline SB8-05dcp-21 & SB8-21dcp-12 & SB8-16dcp-21 & SB8-10dcp-12 & SB8-20dcp-11 & SB8-07dcp-22 \\
\hline Std-dcp-112 & Std-dcp-122 & Std-dcp-212 & Std-dcp-222 & Std-dcp-312 & Std-dcp-322 \\
\hline SB8-19dcp-21 & SB8-19dcp-12 & SB8-17dcp-21 & SB8-16dcp-22 & SB8-14dcp-21 & SB8-15dcp-22 \\
\hline SB8-03dcp-21 & SB8-08dcp-22 & SB8-10dcp-21 & SB8-22dcp-22 & SB8-07dcp-21 & SB8-14dcp-22 \\
\hline SB8-18dcp-21 & SB8-19dcp-22 & SB8-04dcp-21 & SB8-22dcp-12 & SB8-20dcp-21 & SB8-13dcp-22 \\
\hline SB8-09dcp-21 & SB8-18dcp-22 & SB8-01dcp-11 & SB8-11dcp-22 & SB8-14dcp-11 & SB8-02dcp-22 \\
\hline SB8-06dcp-11 & SB8-03dcp-22 & SB8-22dcp-11 & SB8-17dcp-22 & SB8-15dcp-11 & SB8-02dcp-12 \\
\hline SB8-09dcp-11 & SB8-05dcp-12 & SB8-17dcp-11 & SB8-04dcp-22 & SB8-07dcp-11 & SB8-13dcp-12 \\
\hline SB8-08dcp-21 & SB8-21dcp-22 & Std-dcp-213 & Std-dcp-223 & Std-dcp-313 & Std-dcp-323 \\
\hline SB8-05dcp-11 & SB8-09dcp-12 & & & & \\
\hline Std-dcp-113 & Std-dcp-123 & & & & \\
\hline
\end{tabular}

The sample identifiers in Table 6 have been modified to provide a labeling scheme for the measurements that are to be generated by these DCP determinations. Specifically, a suffix has been added to the label for the standard, Std-dcp, to indicate the DCP block (i.e., 1, 2, or 3), the calibration 
set (i.e., 1 (first) or 2 (second)), and its position (i.e., 1, 2, or 3) in the set. Finally, a suffix has been added to the sample identifier that indicates the calibration set (i.e., 1 or 2 ).

Attachment 1 repeats the critical portions and tables supporting the laboratory's activities associated with the chemical composition analysis and reporting. This attachment is intended to provide a "detachable” work plan that may be provided to laboratory technicians.

\subsection{Analytical Plan for the Measurement of PCT Solutions}

A primary property of interest for the glasses of this study is durability as determined by the PCT, which is defined in ASTM C 1285 (latest revision) [2]. As stated in Section 1.0, the study glasses are to be subjected to two heat treatments. During their initial fabrication, the glasses are to be quenched. In a subsequent step, they are to be cooled to simulate cooling along the centerline of a DWPF-type canister. This cooling schedule is referred to as the centerline canister cooling (ccc) curve. Both heat treatments (prepared in triplicate) of each study glass will be subjected to the PCT. Comparisons between the durabilities of the resulting glasses will provide insight into the effects of thermal history on the product performance for these glasses.

Due to the large number of PCTs that are to be performed as part of this study, several oven runs are to be conducted with the study glasses grouped in each of these runs as detailed in this section. In addition to the study glasses, each oven run is to include triplicate PCTs of the Approved Reference Material - One (ARM-1) glass and triplicate PCTs of the EA glass. Two reagent blank samples are also to be included in each oven run. Each oven run is to contain 32 vessels with 8 vessels being needed for the standard and blank solutions. That leaves 24 vessels to support the study glasses in each oven run. With 6 vessels needed for a given study glass ( 3 for the quenched version and 3 for the ccc version), 4 study glasses can be included in each oven run. Thus, 6 oven runs are to be conducted to cover all of the PCTs needed to support this study. The activities involved in the completion of each oven run include labeling and grouping of the PCT solutions and of the solutions of the standards. These solutions are then to be grouped and ordered for analysis by Inductively Coupled Plasma - Atomic Emission Spectroscopy (ICP) or by DCP. While the primary interest of these analyses is the measured concentrations of boron (B), lithium (Li), sodium (Na), and silicon (Si), the measurements of other elements (e.g., aluminum, iron, thorium, uranium, etc.) may also be recorded by VSL in order to provide a more complete set of results for these analyses. The results are to be reported to SRNL in parts per million (ppm). A measurement below its detection limit should be indicated in this table by a less than sign (“<”) followed by the detection limit.

\subsection{Grouping of Glasses for Oven Runs}

Table 7 presents the identifying codes, SB8-pct-001 through SB8-pct-180, that are to be used to label the individual solutions for each of the 6 oven runs that are required to complete these PCTs. Labels are provided for the solutions of the study glasses and of the standards (EA, ARM-1, and blanks). These codes provide a naming convention that is to be established by the VSL technicians conducting the PCT oven runs and then used by the laboratory technicians conducting the ICP or DCP analyses. 
Table 7. Groupings of Glasses for Oven Runs and the Labeling of the Corresponding PCT Solutions

\begin{tabular}{|c|c|c|c|c|c|c|c|c|c|c|c|}
\hline \multicolumn{2}{|c|}{ Oven Run 1} & \multicolumn{2}{|c|}{ Oven Run 2} & \multicolumn{2}{|c|}{ Oven Run 3} & \multicolumn{2}{|c|}{ Oven Run 4} & \multicolumn{2}{|c|}{ Oven Run 5} & \multicolumn{2}{|c|}{ Oven Run 6} \\
\hline Glass ID & Solution ID & Glass ID & Solution ID & Glass ID & Solution ID & Glass ID & Solution ID & Glass ID & Solution ID & Glass ID & Solution ID \\
\hline ARM & SB8-pct-030 & ARM & SB8-pct-116 & ARM & SB8-pct-164 & ARM & SB8-pct-047 & ARM & SB8-pct-119 & ARM & SB8-pct-006 \\
\hline ARM & SB8-pct-178 & ARM & SB8-pct-129 & ARM & SB8-pct-165 & ARM & SB8-pct-142 & ARM & SB8-pct-005 & ARM & SB8-pct-012 \\
\hline ARM & SB8-pct-098 & ARM & SB8-pct-100 & ARM & SB8-pct-007 & ARM & SB8-pct-029 & ARM & SB8-pct-127 & ARM & SB8-pct-177 \\
\hline blank & SB8-pct-032 & blank & SB8-pct-061 & blank & SB8-pct-078 & blank & SB8-pct-063 & blank & SB8-pct-171 & blank & SB8-pct-041 \\
\hline blank & SB8-pct-088 & blank & SB8-pct-170 & blank & SB8-pct-156 & blank & SB8-pct-112 & blank & SB8-pct-034 & blank & SB8-pct-108 \\
\hline EA & SB8-pct-091 & EA & SB8-pct-076 & EA & SB8-pct-013 & EA & SB8-pct-159 & EA & SB8-pct-089 & EA & SB8-pct-102 \\
\hline EA & SB8-pct-056 & EA & SB8-pct-160 & EA & SB8-pct-059 & EA & SB8-pct-172 & EA & SB8-pct-103 & EA & SB8-pct-130 \\
\hline EA & SB8-pct-065 & EA & SB8-pct-135 & EA & SB8-pct-072 & EA & SB8-pct-138 & EA & SB8-pct-141 & EA & SB8-pct-081 \\
\hline SB8-03 & SB8-pct-145 & SB8-06 & SB8-pct-118 & SB8-04 & SB8-pct-095 & SB8-01 & SB8-pct-071 & SB8-02 & SB8-pct-062 & SB8-13 & SB8-pct-019 \\
\hline SB8-03 & SB8-pct-161 & SB8-06 & SB8-pct-153 & SB8-04 & SB8-pct-040 & SB8-01 & SB8-pct-107 & SB8-02 & SB8-pct-044 & SB8-13 & SB8-pct-010 \\
\hline SB8-03 & SB8-pct-051 & SB8-06 & SB8-pct-075 & SB8-04 & SB8-pct-151 & SB8-01 & SB8-pct-093 & SB8-02 & SB8-pct-009 & SB8-13 & SB8-pct-109 \\
\hline SB8-03cсc & SB8-pct-143 & SB8-06ccC & SB8-pct-158 & SB8-04ccC & SB8-pct-121 & SB8-01ccC & SB8-pct-146 & SB8-02ccC & SB8-pct-120 & SB8-13cCC & SB8-pct-152 \\
\hline SB8-03ссс & SB8-pct-169 & SB8-06сcс & SB8-pct-069 & SB8-04ccC & SB8-pct-133 & SB8-01ccc & SB8-pct-039 & SB8-02ccc & SB8-pct-137 & SB8-13сcC & SB8-pct-134 \\
\hline SB8-03сcс & SB8-pct-167 & SB8-06ccC & SB8-pct-068 & SB8-04ccC & SB8-pct-082 & SB8-01ccC & SB8-pct-073 & SB8-02ccC & SB8-pct-154 & SB8-13ccc & SB8-pct-014 \\
\hline SB8-05 & SB8-pct-175 & SB8-08 & SB8-pct-083 & SB8-16 & SB8-pct-157 & SB8-10 & SB8-pct-079 & SB8-07 & SB8-pct-057 & SB8-14 & SB8-pct-084 \\
\hline SB8-05 & SB8-pct-042 & SB8-08 & SB8-pct-001 & SB8-16 & SB8-pct-080 & SB8-10 & SB8-pct-162 & SB8-07 & SB8-pct-043 & SB8-14 & SB8-pct-128 \\
\hline SB8-05 & SB8-pct-094 & SB8-08 & SB8-pct-148 & SB8-16 & SB8-pct-163 & SB8-10 & SB8-pct-147 & SB8-07 & SB8-pct-174 & SB8-14 & SB8-pct-025 \\
\hline SB8-05ccc & SB8-pct-003 & SB8-08ccC & SB8-pct-036 & SB8-16ccC & SB8-pct-070 & SB8-10ccc & SB8-pct-017 & SB8-07ccc & SB8-pct-035 & SB8-14CCC & SB8-pct-028 \\
\hline SB8-05сcC & SB8-pct-110 & SB8-08ccC & SB8-pct-124 & SB8-16сcC & SB8-pct-021 & SB8-10ccC & SB8-pct-144 & SB8-07ccC & SB8-pct-026 & SB8-14CCC & SB8-pct-173 \\
\hline SB8-05сcC & SB8-pct-104 & SB8-08cсc & SB8-pct-140 & SB8-16сcC & SB8-pct-122 & SB8-10сcс & SB8-pct-066 & SB8-07ccc & SB8-pct-046 & SB8-14CCC & SB8-pct-125 \\
\hline SB8-18 & SB8-pct-101 & SB8-09 & SB8-pct-053 & SB8-22 & SB8-pct-058 & SB8-11 & SB8-pct-136 & SB8-12 & SB8-pct-114 & SB8-20 & SB8-pct-023 \\
\hline SB8-18 & SB8-pct-131 & SB8-09 & SB8-pct-092 & SB8-22 & SB8-pct-115 & SB8-11 & SB8-pct-087 & SB8-12 & SB8-pct-048 & SB8-20 & SB8-pct-067 \\
\hline SB8-18 & SB8-pct-022 & SB8-09 & SB8-pct-037 & SB8-22 & SB8-pct-054 & SB8-11 & SB8-pct-024 & SB8-12 & SB8-pct-049 & SB8-20 & SB8-pct-055 \\
\hline SB8-18cсc & SB8-pct-179 & SB8-09cсc & SB8-pct-027 & SB8-22ccC & SB8-pct-099 & SB8-11ccC & SB8-pct-011 & SB8-12ccC & SB8-pct-106 & SB8-20ccC & SB8-pct-096 \\
\hline SB8-18ccc & SB8-pct-113 & SB8-09ccc & SB8-pct-111 & SB8-22cCC & SB8-pct-123 & SB8-11ccc & SB8-pct-018 & SB8-12ccc & SB8-pct-166 & SB8-20ccC & SB8-pct-016 \\
\hline SB8-18сcс & SB8-pct-105 & SB8-09сcс & SB8-pct-132 & SB8-22ccc & SB8-pct-031 & SB8-11ccc & SB8-pct-064 & SB8-12ccC & SB8-pct-004 & SB8-20ccc & SB8-pct-168 \\
\hline SB8-21 & SB8-pct-074 & SB8-19 & SB8-pct-008 & & & SB8-17 & SB8-pct-097 & SB8-15 & SB8-pct-002 & & \\
\hline SB8-21 & SB8-pct-052 & SB8-19 & SB8-pct-050 & & & SB8-17 & SB8-pct-117 & SB8-15 & SB8-pct-038 & & \\
\hline SB8-21 & SB8-pct-060 & SB8-19 & SB8-pct-126 & & & SB8-17 & SB8-pct-155 & SB8-15 & SB8-pct-015 & & \\
\hline SB8-21ccc & SB8-pct-150 & SB8-19ccc & SB8-pct-149 & & & SB8-17ccC & SB8-pct-085 & SB8-15ccc & SB8-pct-176 & & \\
\hline SB8-21ccc & SB8-pct-180 & SB8-19cсc & SB8-pct-139 & & & SB8-17ccC & SB8-pct-090 & SB8-15ccc & SB8-pct-077 & & \\
\hline SB8-21ccc & SB8-pct-086 & SB8-19cсC & SB8-pct-033 & & & SB8-17ccC & SB8-pct-020 & SB8-15ccC & SB8-pct-045 & & \\
\hline
\end{tabular}




\subsection{Measurement of the PCT Solutions}

A multi-element solution standard (denoted by "std-ij" where $\mathrm{i}=\mathrm{A}, \mathrm{B}, \mathrm{C}, \ldots$, I represents one of the nine block letters and $\mathrm{j}=1,2$, and 3 represents the position in the block) is to be added at the beginning, middle, and end of each of the nine ICP or DCP blocks by the VSL laboratory technicians. VSL is to provide SRNL the reference values in ppm for the elemental concentrations of the multielement standard. The measurements of the standard may be useful in checking for bias in the concentration measurements arising from the ICP or DCP calibrations.

Table 8 presents a plan for the leachate measurements for the PCTs. As the analyses are conducted by VSL, each sample group or block of Table 8 requires a different calibration of the ICP or DCP instrumentation. Each of the solution samples of Table 8 is to be analyzed only once for each of the elements of interest to VSL. However, the following elements are to be provided to SRNL for further evaluation: boron (B), lithium ( $\mathrm{Li}$ ), sodium (Na), and silicon ( $\mathrm{Si}$ ) concentrations. The measurements provided to SRNL are to be reported in ppm after accounting for all dilutions and other adjustments conducted during the measurement process.

Attachment 2 repeats the critical portions and tables supporting the laboratory's activities in determining the PCT results and reporting the data to SRNL. This attachment is intended to provide a "detachable” work plan that may be provided to laboratory technicians. 
Table 8. ICP or DCP Calibration Blocks for the Leachate Measurements for the PCTs ${ }^{f}$

\begin{tabular}{|c|c|c|c|c|c|c|c|c|}
\hline \multicolumn{3}{|c|}{ PCT Solutions from Oven Runs 1 and 2} & \multicolumn{3}{|c|}{ PCT Solutions from Oven Runs 3 and 4} & \multicolumn{3}{|c|}{ PCT Solutions from Oven Runs 5 and 6} \\
\hline $\begin{array}{c}\text { Calibration } \\
\text { Block A }\end{array}$ & $\begin{array}{c}\text { Calibration } \\
\text { Block B } \\
\end{array}$ & $\begin{array}{c}\text { Calibration } \\
\text { Block C }\end{array}$ & $\begin{array}{c}\text { Calibration } \\
\text { Block D }\end{array}$ & $\begin{array}{c}\text { Calibration } \\
\text { Block E } \\
\end{array}$ & $\begin{array}{c}\text { Calibration } \\
\text { Block F }\end{array}$ & $\begin{array}{c}\text { Calibration } \\
\text { Block G }\end{array}$ & $\begin{array}{c}\text { Calibration } \\
\text { Block H }\end{array}$ & $\begin{array}{c}\text { Calibration } \\
\text { Block I }\end{array}$ \\
\hline std-A1 & std-B1 & std-C1 & std-D1 & std-E1 & std-F1 & std-G1 & std-H1 & std-I1 \\
\hline SB8-pct-074 & SB8-pct-153 & SB8-pct-060 & SB8-pct-164 & SB8-pct-018 & SB8-pct-151 & SB8-pct-002 & SB8-pct-043 & SB8-pct-009 \\
\hline SB8-pct-116 & SB8-pct-050 & SB8-pct-075 & SB8-pct-011 & SB8-pct-087 & SB8-pct-031 & SB8-pct-028 & SB8-pct-044 & SB8-pct-141 \\
\hline SB8-pct-003 & SB8-pct-042 & SB8-pct-068 & SB8-pct-157 & SB8-pct-080 & SB8-pct-073 & SB8-pct-171 & SB8-pct-137 & SB8-pct-046 \\
\hline SB8-pct-083 & SB8-pct-092 & SB8-pct-135 & SB8-pct-159 & SB8-pct-107 & SB8-pct-054 & SB8-pct-019 & SB8-pct-041 & SB8-pct-049 \\
\hline SB8-pct-053 & SB8-pct-056 & SB8-pct-105 & SB8-pct-070 & SB8-pct-063 & SB8-pct-093 & SB8-pct-096 & SB8-pct-128 & SB8-pct-125 \\
\hline SB8-pct-101 & SB8-pct-124 & SB8-pct-104 & SB8-pct-047 & SB8-pct-133 & SB8-pct-072 & SB8-pct-057 & SB8-pct-134 & SB8-pct-025 \\
\hline SB8-pct-076 & SB8-pct-052 & SB8-pct-051 & SB8-pct-013 & SB8-pct-142 & SB8-pct-024 & SB8-pct-062 & SB8-pct-026 & SB8-pct-154 \\
\hline SB8-pct-091 & SB8-pct-110 & SB8-pct-100 & SB8-pct-017 & SB8-pct-172 & SB8-pct-163 & SB8-pct-089 & SB8-pct-166 & SB8-pct-081 \\
\hline SB8-pct-030 & SB8-pct-001 & SB8-pct-132 & SB8-pct-085 & SB8-pct-040 & SB8-pct-082 & SB8-pct-152 & SB8-pct-038 & SB8-pct-174 \\
\hline SB8-pct-008 & SB8-pct-169 & SB8-pct-126 & SB8-pct-099 & SB8-pct-123 & SB8-pct-029 & SB8-pct-114 & SB8-pct-048 & SB8-pct-015 \\
\hline SB8-pct-118 & SB8-pct-178 & SB8-pct-098 & SB8-pct-095 & SB8-pct-165 & SB8-pct-020 & SB8-pct-035 & SB8-pct-005 & SB8-pct-108 \\
\hline std-A2 & std-B2 & std-C2 & std-D2 & std-E2 & std-F2 & std-G2 & std-H2 & std-I2 \\
\hline SB8-pct-149 & SB8-pct-160 & SB8-pct-086 & SB8-pct-146 & SB8-pct-021 & SB8-pct-138 & SB8-pct-102 & SB8-pct-077 & SB8-pct-177 \\
\hline SB8-pct-150 & SB8-pct-088 & SB8-pct-094 & SB8-pct-121 & SB8-pct-090 & SB8-pct-147 & SB8-pct-176 & SB8-pct-012 & SB8-pct-004 \\
\hline SB8-pct-027 & SB8-pct-129 & SB8-pct-022 & SB8-pct-097 & SB8-pct-117 & SB8-pct-007 & SB8-pct-023 & SB8-pct-130 & SB8-pct-168 \\
\hline SB8-pct-158 & SB8-pct-061 & SB8-pct-065 & SB8-pct-136 & SB8-pct-115 & SB8-pct-112 & SB8-pct-119 & SB8-pct-010 & SB8-pct-055 \\
\hline SB8-pct-032 & SB8-pct-180 & SB8-pct-037 & SB8-pct-071 & SB8-pct-156 & SB8-pct-064 & SB8-pct-106 & SB8-pct-173 & SB8-pct-045 \\
\hline SB8-pct-036 & SB8-pct-139 & SB8-pct-140 & SB8-pct-079 & SB8-pct-039 & SB8-pct-122 & SB8-pct-120 & SB8-pct-067 & SB8-pct-127 \\
\hline SB8-pct-143 & SB8-pct-113 & SB8-pct-167 & SB8-pct-078 & SB8-pct-059 & SB8-pct-155 & SB8-pct-006 & SB8-pct-103 & SB8-pct-014 \\
\hline SB8-pct-179 & SB8-pct-161 & SB8-pct-148 & SB8-pct-058 & SB8-pct-144 & SB8-pct-066 & SB8-pct-084 & SB8-pct-034 & SB8-pct-109 \\
\hline SB8-pct-145 & SB8-pct-069 & SB8-pct-170 & std-D3 & SB8-pct-162 & std-F3 & std-G3 & SB8-pct-016 & std-I3 \\
\hline SB8-pct-175 & SB8-pct-131 & SB8-pct-033 & & std-E3 & & & std-H3 & \\
\hline \multirow[t]{2}{*}{ std-A3 } & SB8-pct-111 & std-C3 & & & & & & \\
\hline & std-B3 & & & & & & & \\
\hline
\end{tabular}

${ }^{f}$ Note that the PCT solutions from two oven runs are needed to support each set of 3 blocks: A-C, D-F, and G-I. 
SRNL-STI-2012-00725

Revision 0

\subsection{SUMMARY}

ES/VSL are to provide engineering and technical services support to SRR for ongoing operation of the DWPF flowsheet as well as for modifications to improve overall plant performance. SRR has requested via a SOW that ES/VSL conduct a glass variability study for SB8. SRR issued a TTR requesting that SRNL provide planning and data reduction support for the ES/VSL effort. This document provides two analytical plans for use by ES/VSL: one plan is to guide the measurement of the chemical composition of the study glasses while the second is to guide the measurement of the durability of the study glasses based upon their PCT responses. The measurements generated by ES/VSL are to be provided to SRNL for data reduction and evaluation. SRNL is to review these results with ES/VSL and SRR, and the results are to be incorporated into a joint report with ES/VSL as a deliverable to SRR to support the processing of SB8 at DWPF.

\subsection{REFERENCES}

[1] Fellinger, T.L., "Sludge Batch 7A Variability Study and Glass Property Measurements,” XSOW-S-00003, Revision 2, Savannah River Remediation, Aiken SC, September 2012.

[2] ASTM International, "Standard Test Methods for Determining Chemical Durability of Nuclear, Hazardous, and Mixed Waste Glasses and Multiphase Glass Ceramics: The Product Consistency Test (PCT),” ASTM International, West Conshohocken, PA, ASTM C 1285-02, 2002.

[3] Samadi, A., “Sludge Batch 8 Frit Optimization,” HLW-DWPF-TTR-2012-0011, Revision 0, 2012.

[4] Peeler, D.K. and T.B. Edwards, “Task Technical and Quality Assurance Plan for Sludge Batch 8 Frit Optimization,” SRNL-RP-2012-00070, July 17, 2012. 


\section{Attachment 1.}

\section{Sample Preparation and Measurement of Glass Chemical Compositions ${ }^{f}$}

Measurements for the following elements of interest are to be acquired by VSL using X-ray Fluorescence Spectroscopy (XRF): aluminum (Al), barium (Ba), calcium (Ca), cerium (Ce), chromium $(\mathrm{Cr})$, copper $(\mathrm{Cu})$, iron $(\mathrm{Fe})$, potassium $(\mathrm{K})$, lanthanum $(\mathrm{La})$, magnesium $(\mathrm{Mg})$, manganese $(\mathrm{Mn})$, sodium $(\mathrm{Na})$, nickel $(\mathrm{Ni})$, lead $(\mathrm{Pb})$, sulfur $(\mathrm{S})$, silicon $(\mathrm{Si})$, thorium $(\mathrm{Th})$, titanium $(\mathrm{Ti})$, uranium (U), zinc ( $\mathrm{Zn})$, and zirconium ( $\mathrm{Zr}$ ) concentrations. It should be noted that some of the elements listed above are minor components of the study glasses and may be below the detection limits of the analytical procedure.

Table A1.1 presents the XRF measurement blocks. Also, a single sample of a reference glass, that is to be selected by VSL to be a matrix-match for this study and that is to be labeled as "Std-xrf", is to be submitted repeatedly for measurement along with the samples of the study glasses as indicated in Table A1.1. These compositions are to be provided to SRNL as weight percent (wt\%) elemental concentrations. Table A1.2 provides a template for use by VSL in reporting these results. A value below its detection limit should be indicated in this table by a less than sign ("<”) followed by the detection limit.

Table A1.1 XRF Analytical Blocks and Sequencing along with the Measurement Identifiers

\begin{tabular}{|c|c|c|c|c|c|}
\hline $\begin{array}{c}\text { XRF Block } 1 \\
\text { First Set of } \\
\text { Measurements }\end{array}$ & $\begin{array}{l}\text { XRF Block } 1 \\
\text { Second Set of } \\
\text { Measurements }\end{array}$ & $\begin{array}{c}\text { XRF Block } 2 \\
\text { First Set of } \\
\text { Measurements }\end{array}$ & $\begin{array}{c}\text { XRF Block } 2 \\
\text { Second Set of } \\
\text { Measurements }\end{array}$ & $\begin{array}{c}\text { XRF Block } 3 \\
\text { First Set of } \\
\text { Measurements }\end{array}$ & $\begin{array}{c}\text { XRF Block } 3 \\
\text { Second Set of } \\
\text { Measurements }\end{array}$ \\
\hline Std-xrf-111 & Std-xrf-121 & Std-xrf-211 & Std-xrf-221 & Std-xrf-311 & Std-xrf-321 \\
\hline SB8-21xrf-11 & SB8-09xrf-22 & SB8-16xrf-11 & SB8-17xrf-12 & SB8-12xrf-11 & SB8-20xrf-22 \\
\hline SB8-19xrf-11 & SB8-08xrf-12 & SB8-04xrf-11 & SB8-04xrf-12 & SB8-15xrf-21 & SB8-12xrf-12 \\
\hline SB8-08xrf-11 & SB8-05xrf-22 & SB8-11xrf-11 & SB8-11xrf-12 & SB8-12xrf-21 & SB8-20xrf-12 \\
\hline SB8-03xrf-11 & SB8-03xrf-12 & SB8-11xrf-21 & SB8-10xrf-22 & SB8-13xrf-11 & SB8-12xrf-22 \\
\hline SB8-18xrf-11 & SB8-06xrf-12 & SB8-22xrf-21 & SB8-16xrf-12 & SB8-02xrf-21 & SB8-15xrf-12 \\
\hline SB8-06xrf-21 & SB8-06xrf-22 & SB8-10xrf-11 & SB8-01xrf-22 & SB8-13xrf-21 & SB8-07xrf-12 \\
\hline SB8-21xrf-21 & SB8-18xrf-12 & SB8-16xrf-21 & SB8-01xrf-12 & SB8-02xrf-11 & SB8-14xrf-12 \\
\hline SB8-05xrf-21 & SB8-21xrf-12 & SB8-01xrf-21 & SB8-10xrf-12 & SB8-20xrf-11 & SB8-07xrf-22 \\
\hline Std-xrf-112 & Std-xrf-122 & Std-xrf-212 & Std-xrf-222 & Std-xrf-312 & Std-xrf-322 \\
\hline SB8-19xrf-21 & SB8-19xrf-12 & SB8-17xrf-21 & SB8-16xrf-22 & SB8-14xrf-21 & SB8-15xrf-22 \\
\hline SB8-03xrf-21 & SB8-08xrf-22 & SB8-10xrf-21 & SB8-22xrf-22 & SB8-07xrf-21 & SB8-14xrf-22 \\
\hline SB8-18xrf-21 & SB8-19xrf-22 & SB8-04xrf-21 & SB8-22xrf-12 & SB8-20xrf-21 & SB8-13xrf-22 \\
\hline SB8-09xrf-21 & SB8-18xrf-22 & SB8-01xrf-11 & SB8-11xrf-22 & SB8-14xrf-11 & SB8-02xrf-22 \\
\hline SB8-06xrf-11 & SB8-03xrf-22 & SB8-22xrf-11 & SB8-17xrf-22 & SB8-15xrf-11 & SB8-02xrf-12 \\
\hline SB8-09xrf-11 & SB8-05xrf-12 & SB8-17xrf-11 & SB8-04xrf-22 & SB8-07xrf-11 & SB8-13xrf-12 \\
\hline SB8-08xrf-21 & SB8-21xrf-22 & Std-xrf-213 & Std-xrf-223 & Std-xrf-313 & Std-xrf-323 \\
\hline SB8-05xrf-11 & SB8-09xrf-12 & & & & \\
\hline Std-xrf-113 & Std-xrf-123 & & & & \\
\hline
\end{tabular}

\footnotetext{
${ }^{f}$ Copies of this attachment may be provided to the laboratory technicians at VSL who are to conduct the sample dissolutions required to prepare the glass samples for DCP analysis, to those who are to conduct the XRF analyses, and to those that are to conduct the DCP analyses to facilitate these activities.
} 
Table A1.2 Template for Reporting XRF Results

\begin{tabular}{|c|c|c|c|c|c|c|c|c|c|c|c|c|c|c|c|c|c|c|c|c|c|c|c|}
\hline $\begin{array}{c}\text { Block } \\
\text { Number }\end{array}$ & $\begin{array}{c}\text { Measurement } \\
\text { Group }\end{array}$ & Sample ID & Al & Ba & Ca & Ce & $\mathrm{Cr}$ & $\mathbf{C u}$ & $\mathbf{F e}$ & $\mathbf{K}$ & $\mathbf{L a}$ & Mg & Mn & $\mathrm{Na}$ & $\mathbf{N i}$ & $\mathbf{P b}$ & $\mathbf{S}$ & Si & Th & $\mathbf{T i}$ & $\mathbf{U}$ & Zn & $\mathrm{Zr}$ \\
\hline 1 & 1 & Std-xrf-111 & & & & & & & & & & & & & & & & & & & & & \\
\hline 1 & 1 & SB8-21xrf-11 & & & & & & & & & & & & & & & & & & & & & \\
\hline 1 & 1 & SB8-19xrf-11 & & & & & & & & & & & & & & & & & & & & & \\
\hline 1 & 1 & SB8-08xrf-11 & & & & & & & & & & & & & & & & & & & & & \\
\hline 1 & 1 & SB8-03xrf-11 & & & & & & & & & & & & & & & & & & & & & \\
\hline 1 & 1 & SB8-18xrf-11 & & & & & & & & & & & & & & & & & & & & & \\
\hline 1 & 1 & SB8-06xrf-21 & & & & & & & & & & & & & & & & & & & & & \\
\hline 1 & 1 & SB8-21xrf-21 & & & & & & & & & & & & & & & & & & & & & \\
\hline 1 & 1 & SB8-05xrf-21 & & & & & & & & & & & & & & & & & & & & & \\
\hline 1 & 1 & Std-xrf-112 & & & & & & & & & & & & & & & & & & & & & \\
\hline 1 & 1 & SB8-19xrf-21 & & & & & & & & & & & & & & & & & & & & & \\
\hline 1 & 1 & SB8-03xrf-21 & & & & & & & & & & & & & & & & & & & & & \\
\hline 1 & 1 & SB8-18xrf-21 & & & & & & & & & & & & & & & & & & & & & \\
\hline 1 & 1 & SB8-09xrf-21 & & & & & & & & & & & & & & & & & & & & & \\
\hline 1 & 1 & SB8-06xrf-11 & & & & & & & & & & & & & & & & & & & & & \\
\hline 1 & 1 & SB8-09xrf-11 & & & & & & & & & & & & & & & & & & & & & \\
\hline 1 & 1 & SB8-08xrf-21 & & & & & & & & & & & & & & & & & & & & & \\
\hline 1 & 1 & SB8-05xrf-11 & & & & & & & & & & & & & & & & & & & & & \\
\hline 1 & 1 & Std-xrf-113 & & & & & & & & & & & & & & & & & & & & & \\
\hline 1 & 2 & Std-xrf-121 & & & & & & & & & & & & & & & & & & & & & \\
\hline 1 & 2 & SB8-09xrf-22 & & & & & & & & & & & & & & & & & & & & & \\
\hline 1 & 2 & SB8-08xrf-12 & & & & & & & & & & & & & & & & & & & & & \\
\hline 1 & 2 & SB8-05xrf-22 & & & & & & & & & & & & & & & & & & & & & \\
\hline 1 & 2 & SB8-03xrf-12 & & & & & & & & & & & & & & & & & & & & & \\
\hline 1 & 2 & SB8-06xrf-12 & & & & & & & & & & & & & & & & & & & & & \\
\hline 1 & 2 & SB8-06xrf-22 & & & & & & & & & & & & & & & & & & & & & \\
\hline 1 & 2 & SB8-18xrf-12 & & & & & & & & & & & & & & & & & & & & & \\
\hline 1 & 2 & SB8-21xrf-12 & & & & & & & & & & & & & & & & & & & & & \\
\hline 1 & 2 & Std-xrf-122 & & & & & & & & & & & & & & & & & & & & & \\
\hline 1 & 2 & SB8-19xrf-12 & & & & & & & & & & & & & & & & & & & & & \\
\hline 1 & 2 & SB8-08xrf-22 & & & & & & & & & & & & & & & & & & & & & \\
\hline 1 & 2 & SB8-19xrf-22 & & & & & & & & & & & & & & & & & & & & & \\
\hline 1 & 2 & SB8-18xrf-22 & & & & & & & & & & & & & & & & & & & & & \\
\hline 1 & 2 & SB8-03xrf-22 & & & & & & & & & & & & & & & & & & & & & \\
\hline 1 & 2 & SB8-05xrf-12 & & & & & & & & & & & & & & & & & & & & & \\
\hline 1 & 2 & SB8-21xrf-22 & & & & & & & & & & & & & & & & & & & & & \\
\hline 1 & 2 & SB8-09xrf-12 & & & & & & & & & & & & & & & & & & & & & \\
\hline 1 & 2 & Std-xrf-123 & & & & & & & & & & & & & & & & & & & & & \\
\hline 2 & 1 & Std-xrf-211 & & & & & & & & & & & & & & & & & & & & & \\
\hline 2 & 1 & SB8-16xrf-11 & & & & & & & & & & & & & & & & & & & & & \\
\hline 2 & 1 & SB8-04xrf-11 & & & & & & & & & & & & & & & & & & & & & \\
\hline
\end{tabular}




\begin{tabular}{|c|c|c|c|c|c|c|c|c|c|c|c|c|c|c|c|c|c|c|c|c|c|c|c|}
\hline $\begin{array}{c}\text { Block } \\
\text { Number }\end{array}$ & $\begin{array}{l}\text { Measurement } \\
\text { Group }\end{array}$ & Sample ID & Al & Ba & Ca & Ce & $\mathbf{C r}$ & $\mathrm{Cu}$ & $\mathbf{F e}$ & $\mathbf{K}$ & La & Mg & Mn & $\mathrm{Na}$ & $\mathrm{Ni}$ & $\mathbf{P b}$ & $\mathbf{S}$ & $\mathrm{Si}$ & Th & $\mathbf{T i}$ & $\mathbf{U}$ & Zn & $\mathrm{Zr}$ \\
\hline 2 & 1 & SB8-11xrf-11 & & & & & & & & & & & & & & & & & & & & & \\
\hline 2 & 1 & SB8-11xrf-21 & & & & & & & & & & & & & & & & & & & & & \\
\hline 2 & 1 & SB8-22xrf-21 & & & & & & & & & & & & & & & & & & & & & \\
\hline 2 & 1 & SB8-10xrf-11 & & & & & & & & & & & & & & & & & & & & & \\
\hline 2 & 1 & SB8-16xrf-21 & & & & & & & & & & & & & & & & & & & & & \\
\hline 2 & 1 & SB8-01xrf-21 & & & & & & & & & & & & & & & & & & & & & \\
\hline 2 & 1 & Std-xrf-212 & & & & & & & & & & & & & & & & & & & & & \\
\hline 2 & 1 & SB8-17xrf-21 & & & & & & & & & & & & & & & & & & & & & \\
\hline 2 & 1 & SB8-10xrf-21 & & & & & & & & & & & & & & & & & & & & & \\
\hline 2 & 1 & SB8-04xrf-21 & & & & & & & & & & & & & & & & & & & & & \\
\hline 2 & 1 & SB8-01xrf-11 & & & & & & & & & & & & & & & & & & & & & \\
\hline 2 & 1 & SB8-22xrf-11 & & & & & & & & & & & & & & & & & & & & & \\
\hline 2 & 1 & SB8-17xrf-11 & & & & & & & & & & & & & & & & & & & & & \\
\hline 2 & 1 & Std-xrf-213 & & & & & & & & & & & & & & & & & & & & & \\
\hline 2 & 2 & Std-xrf-221 & & & & & & & & & & & & & & & & & & & & & \\
\hline 2 & 2 & SB8-17xrf-12 & & & & & & & & & & & & & & & & & & & & & \\
\hline 2 & 2 & SB8-04xrf-12 & & & & & & & & & & & & & & & & & & & & & \\
\hline 2 & 2 & SB8-11xrf-12 & & & & & & & & & & & & & & & & & & & & & \\
\hline 2 & 2 & SB8-10xrf-22 & & & & & & & & & & & & & & & & & & & & & \\
\hline 2 & 2 & SB8-16xrf-12 & & & & & & & & & & & & & & & & & & & & & \\
\hline 2 & 2 & SB8-01xrf-22 & & & & & & & & & & & & & & & & & & & & & \\
\hline 2 & 2 & SB8-01xrf-12 & & & & & & & & & & & & & & & & & & & & & \\
\hline 2 & 2 & SB8-10xrf-12 & & & & & & & & & & & & & & & & & & & & & \\
\hline 2 & 2 & Std-xrf-222 & & & & & & & & & & & & & & & & & & & & & \\
\hline 2 & 2 & SB8-16xrf-22 & & & & & & & & & & & & & & & & & & & & & \\
\hline 2 & 2 & SB8-22xrf-22 & & & & & & & & & & & & & & & & & & & & & \\
\hline 2 & 2 & SB8-22xrf-12 & & & & & & & & & & & & & & & & & & & & & \\
\hline 2 & 2 & SB8-11xrf-22 & & & & & & & & & & & & & & & & & & & & & \\
\hline 2 & 2 & SB8-17xrf-22 & & & & & & & & & & & & & & & & & & & & & \\
\hline 2 & 2 & SB8-04xrf-22 & & & & & & & & & & & & & & & & & & & & & \\
\hline 2 & 2 & Std-xrf-223 & & & & & & & & & & & & & & & & & & & & & \\
\hline 3 & 1 & Std-xrf-311 & & & & & & & & & & & & & & & & & & & & & \\
\hline 3 & 1 & SB8-12xrf-11 & & & & & & & & & & & & & & & & & & & & & \\
\hline 3 & 1 & SB8-15xrf-21 & & & & & & & & & & & & & & & & & & & & & \\
\hline 3 & 1 & SB8-12xrf-21 & & & & & & & & & & & & & & & & & & & & & \\
\hline 3 & 1 & SB8-13xrf-11 & & & & & & & & & & & & & & & & & & & & & \\
\hline 3 & 1 & SB8-02xrf-21 & & & & & & & & & & & & & & & & & & & & & \\
\hline 3 & 1 & SB8-13xrf-21 & & & & & & & & & & & & & & & & & & & & & \\
\hline 3 & 1 & SB8-02xrf-11 & & & & & & & & & & & & & & & & & & & & & \\
\hline 3 & 1 & SB8-20xrf-11 & & & & & & & & & & & & & & & & & & & & & \\
\hline 3 & 1 & Std-xrf-312 & & & & & & & & & & & & & & & & & & & & & \\
\hline 3 & 1 & SB8-14xrf-21 & & & & & & & & & & & & & & & & & & & & & \\
\hline 3 & 1 & SB8-07xrf-21 & & & & & & & & & & & & & & & & & & & & & \\
\hline 3 & 1 & SB8-20xrf-21 & & & & & & & & & & & & & & & & & & & & & \\
\hline
\end{tabular}




\begin{tabular}{|c|c|c|c|c|c|c|c|c|c|c|c|c|c|c|c|c|c|c|c|c|c|c|c|}
\hline $\begin{array}{c}\text { Block } \\
\text { Number }\end{array}$ & $\begin{array}{c}\text { Measurement } \\
\text { Group }\end{array}$ & Sample ID & Al & $\mathbf{B a}$ & Ca & Ce & $\mathrm{Cr}$ & $\mathbf{C u}$ & $\mathbf{F e}$ & $\mathbf{K}$ & $\mathbf{L a}$ & Mg & Mn & $\mathrm{Na}$ & $\mathbf{N i}$ & $\mathbf{P b}$ & $\mathrm{S}$ & $\mathrm{Si}$ & Th & $\mathbf{T i}$ & $\mathbf{U}$ & $\mathbf{Z n}$ & $\mathrm{Zr}$ \\
\hline 3 & 1 & SB8-14xrf-11 & & & & & & & & & & & & & & & & & & & & & \\
\hline 3 & 1 & SB8-15xrf-11 & & & & & & & & & & & & & & & & & & & & & \\
\hline 3 & 1 & SB8-07xrf-11 & & & & & & & & & & & & & & & & & & & & & \\
\hline 3 & 1 & Std-xrf-313 & & & & & & & & & & & & & & & & & & & & & \\
\hline 3 & 2 & Std-xrf-321 & & & & & & & & & & & & & & & & & & & & & \\
\hline 3 & 2 & SB8-20xrf-22 & & & & & & & & & & & & & & & & & & & & & \\
\hline 3 & 2 & SB8-12xrf-12 & & & & & & & & & & & & & & & & & & & & & \\
\hline 3 & 2 & SB8-20xrf-12 & & & & & & & & & & & & & & & & & & & & & \\
\hline 3 & 2 & SB8-12xrf-22 & & & & & & & & & & & & & & & & & & & & & \\
\hline 3 & 2 & SB8-15xrf-12 & & & & & & & & & & & & & & & & & & & & & \\
\hline 3 & 2 & SB8-07xrf-12 & & & & & & & & & & & & & & & & & & & & & \\
\hline 3 & 2 & SB8-14xrf-12 & & & & & & & & & & & & & & & & & & & & & \\
\hline 3 & 2 & SB8-07xrf-22 & & & & & & & & & & & & & & & & & & & & & \\
\hline 3 & 2 & Std-xrf-322 & & & & & & & & & & & & & & & & & & & & & \\
\hline 3 & 2 & SB8-15xrf-22 & & & & & & & & & & & & & & & & & & & & & \\
\hline 3 & 2 & SB8-14xrf-22 & & & & & & & & & & & & & & & & & & & & & \\
\hline 3 & 2 & SB8-13xrf-22 & & & & & & & & & & & & & & & & & & & & & \\
\hline 3 & 2 & SB8-02xrf-22 & & & & & & & & & & & & & & & & & & & & & \\
\hline 3 & 2 & SB8-02xrf-12 & & & & & & & & & & & & & & & & & & & & & \\
\hline 3 & 2 & SB8-13xrf-12 & & & & & & & & & & & & & & & & & & & & & \\
\hline 3 & 2 & Std-xrf-323 & & & & & & & & & & & & & & & & & & & & & \\
\hline
\end{tabular}


Table A1.3 presents the DCP preparation blocks. Duplicate samples of each of the study glass vials are to be subjected to microwave-assisted total acid dissolution in Teflon vessels according to VSL standard operating procedures. Typically, a mixture of concentrated $\mathrm{HF}: \mathrm{HNO}_{3}$ is used to conduct the digestions with each digestion leading to a $50 \mathrm{ml}$ solution. The resulting solutions are further diluted to $200 \mathrm{ml}$ before DCP analysis. The $200 \mathrm{ml}$ solutions are to be labeled as indicated in Table A1.3. The groupings of Table A1.3 are established in a manner that indicates that all of the batching and preliminary preparations (i.e., grinding) of the glasses are to be completed before the dissolution activities of Table A1.3 are initiated. Each block of Table A1.3 represents a microwave batch and the sequence provides an order for sample weighing. In addition to the study glasses, a sample of the standard reference glass selected by VSL to support this study is to be similarly prepared in a large enough quantity to allow aliquots of the resulting solution to be submitted for DCP analysis (see Table A1.4). This standard glass (Std-dcp) has been included in each preparation block of Table A1.3.

Table A1.3 DCP Preparation Blocks and Sequencing along with Sample Identifiers for the $200 \mathrm{ml}$ Solutions

\begin{tabular}{|c||c||}
\hline DCP Preparation Block 1 & DCP Preparation Block 2 \\
\hline SB8-07dcp-1 & SB8-05dcp-1 \\
\hline SB8-13dcp-1 & SB8-05dcp-2 \\
\hline SB8-07dcp-2 & SB8-04dcp-1 \\
\hline SB8-15dcp-1 & SB8-19dcp-1 \\
\hline SB8-16dcp-1 & SB8-04dcp-2 \\
\hline SB8-15dcp-2 & SB8-19dcp-2 \\
\hline SB8-17dcp-1 & SB8-10dcp-1 \\
\hline SB8-13dcp-2 & SB8-22dcp-1 \\
\hline SB8-14dcp-1 & Std-dcp \\
\hline SB8-16dcp-2 & SB8-08dcp-1 \\
\hline SB8-06dcp-1 & SB8-09dcp-1 \\
\hline Std-dcp & SB8-09dcp-2 \\
\hline SB8-02dcp-1 & SB8-18dcp-1 \\
\hline SB8-03dcp-1 & SB8-22dcp-2 \\
\hline SB8-21dcp-1 & SB8-12dcp-1 \\
\hline SB8-01dcp-1 & SB8-08dcp-2 \\
\hline SB8-14dcp-2 & SB8-11dcp-1 \\
\hline SB8-17dcp-2 & SB8-10dcp-2 \\
\hline SB8-03dcp-2 & SB8-12dcp-2 \\
\hline SB8-21dcp-2 & SB8-18dcp-2 \\
\hline SB8-06dcp-2 & SB8-11dcp-2 \\
\hline SB8-20dcp-1 & \\
\hline SB8-02dcp-2 & \\
\hline SB8-20dcp-2 & \\
\hline SB8-01dcp-2 & \\
\hline & \\
\hline & \\
\hline
\end{tabular}


The samples prepared for chemical analysis by DCP are to be measured for their boron and lithium content. Each of the duplicate preparations of each study glass is to be measured twice by DCP, with a re-calibration of the instrument being performed between the two measurements. The groupings and sequencing of these samples for DCP analysis is provided in Table A1.4. To repeat, each column of this table indicates a different calibration of the DCP instrumentation. In addition, the order of the samples in a column is the sequence in which the samples should be measured by DCP. Also, note the addition of aliquots of the prepared reference glass, Std-dcp, to these DCP analytical blocks. The "known" composition of this reference glass is to be provided to SRNL along with the measured compositions from Table A1.4. These compositions are to be provided as weight percent (wt\%) elemental concentrations. Table A1.5 provides a template for use by VSL in reporting the results from these DCP analyses. A value below its detection limit should be indicated in this table by a less than sign (“<”) followed by the detection limit.

\section{Table A1.4 DCP Analytical Blocks and Sequencing along with the Measurement Identifiers}

\begin{tabular}{|c|c|c|c|c|c|}
\hline $\begin{array}{c}\text { DCP Block } 1 \\
\text { First } \\
\text { Calibration } \\
\end{array}$ & $\begin{array}{c}\text { DCP Block } 1 \\
\text { Second } \\
\text { Calibration } \\
\end{array}$ & $\begin{array}{c}\text { DCP Block } 2 \\
\text { First } \\
\text { Calibration } \\
\end{array}$ & $\begin{array}{c}\text { DCP Block } 2 \\
\text { Second } \\
\text { Calibration } \\
\end{array}$ & $\begin{array}{c}\text { DCP Block } 3 \\
\text { First } \\
\text { Calibration } \\
\end{array}$ & $\begin{array}{c}\text { DCP Block } 3 \\
\text { Second } \\
\text { Calibration } \\
\end{array}$ \\
\hline Std-dcp-111 & Std-dcp-121 & Std-dcp-211 & Std-dcp-221 & Std-dcp-311 & Std-dcp-321 \\
\hline SB8-21dcp-11 & SB8-09dcp-22 & SB8-01dcp-21 & SB8-11dcp-12 & SB8-12dcp-11 & SB8-20dcp-22 \\
\hline SB8-19dcp-11 & SB8-08dcp-12 & SB8-16dcp-11 & SB8-17dcp-12 & SB8-15dcp-21 & SB8-12dcp-12 \\
\hline SB8-08dcp-11 & SB8-05dcp-22 & SB8-04dcp-11 & SB8-04dcp-12 & SB8-12dcp-21 & SB8-20dcp-12 \\
\hline SB8-03dcp-11 & SB8-03dcp-12 & SB8-11dcp-11 & SB8-10dcp-22 & SB8-13dcp-11 & SB8-12dcp-22 \\
\hline SB8-18dcp-11 & SB8-06dcp-12 & SB8-11dcp-21 & SB8-16dcp-12 & SB8-02dcp-21 & SB8-15dcp-12 \\
\hline SB8-06dcp-21 & SB8-06dсp-22 & SB8-22dcp-21 & SB8-01dcp-22 & SB8-13dcp-21 & SB8-07dcp-12 \\
\hline SB8-21dcp-21 & SB8-18dcp-12 & SB8-10dcp-11 & SB8-01dcp-12 & SB8-02dcp-11 & SB8-14dcp-12 \\
\hline SB8-05dcp-21 & SB8-21dcp-12 & SB8-16dcp-21 & SB8-10dcp-12 & SB8-20dcp-11 & SB8-07dcp-22 \\
\hline Std-dcp-112 & Std-dcp-122 & Std-dcp-212 & Std-dcp-222 & Std-dcp-312 & Std-dcp-322 \\
\hline SB8-19dcp-21 & SB8-19dcp-12 & SB8-17dcp-21 & SB8-16dcp-22 & SB8-14dcp-21 & SB8-15dcp-22 \\
\hline SB8-03dcp-21 & SB8-08dcp-22 & SB8-10dcp-21 & SB8-22dcp-22 & SB8-07dcp-21 & SB8-14dcp-22 \\
\hline SB8-18dcp-21 & SB8-19dcp-22 & SB8-04dcp-21 & SB8-22dcp-12 & SB8-20dcp-21 & SB8-13dср-22 \\
\hline SB8-09dcp-21 & SB8-18dcp-22 & SB8-01dcp-11 & SB8-11dcp-22 & SB8-14dcp-11 & SB8-02dcp-22 \\
\hline SB8-06dcp-11 & SB8-03dcp-22 & SB8-22dcp-11 & SB8-17dcp-22 & SB8-15dcp-11 & SB8-02dcp-12 \\
\hline SB8-09dcp-11 & SB8-05dcp-12 & SB8-17dcp-11 & SB8-04dcp-22 & SB8-07dcp-11 & SB8-13dcp-12 \\
\hline SB8-08dcp-21 & SB8-21dcp-22 & Std-dcp-213 & Std-dcp-223 & Std-dcp-313 & Std-dcp-323 \\
\hline SB8-05dcp-11 & SB8-09dcp-12 & & & & \\
\hline Std-dcp-113 & Std-dcp-123 & & & & \\
\hline
\end{tabular}


Table A1.5 Template for Reporting DCP Results

\begin{tabular}{|c|c|c|c|c|}
\hline $\begin{array}{c}\text { Block } \\
\text { Number }\end{array}$ & $\begin{array}{c}\text { Measurement } \\
\text { Group }\end{array}$ & Sample ID & B & $\mathbf{L i}$ \\
\hline 1 & 1 & Std-dcp-111 & & \\
\hline 1 & 1 & SB8-21dcp-11 & & \\
\hline 1 & 1 & SB8-19dcp-11 & & \\
\hline 1 & 1 & SB8-08dcp-11 & & \\
\hline 1 & 1 & SB8-03dcp-11 & & \\
\hline 1 & 1 & SB8-18dcp-11 & & \\
\hline 1 & 1 & SB8-06dcp-21 & & \\
\hline 1 & 1 & SB8-21dcp-21 & & \\
\hline 1 & 1 & SB8-05dcp-21 & & \\
\hline 1 & 1 & Std-dcp-112 & & \\
\hline 1 & 1 & SB8-19dcp-21 & & \\
\hline 1 & 1 & SB8-03dcp-21 & & \\
\hline 1 & 1 & SB8-18dcp-21 & & \\
\hline 1 & 1 & SB8-09dcp-21 & & \\
\hline 1 & 1 & SB8-06dcp-11 & & \\
\hline 1 & 1 & SB8-09dcp-11 & & \\
\hline 1 & 1 & SB8-08dcp-21 & & \\
\hline 1 & 1 & SB8-05dcp-11 & & \\
\hline 1 & 1 & Std-dcp-113 & & \\
\hline 1 & 2 & Std-dcp-121 & & \\
\hline 1 & 2 & SB8-09dcp-22 & & \\
\hline 1 & 2 & SB8-08dcp-12 & & \\
\hline 1 & 2 & SB8-05dcp-22 & & \\
\hline 1 & 2 & SB8-03dcp-12 & & \\
\hline 1 & 2 & SB8-06dcp-12 & & \\
\hline 1 & 2 & SB8-06dcp-22 & & \\
\hline 1 & 2 & SB8-18dcp-12 & & \\
\hline 1 & 2 & SB8-21dcp-12 & & \\
\hline 1 & 2 & Std-dcp-122 & & \\
\hline 1 & 2 & SB8-19dcp-12 & & \\
\hline 1 & 2 & SB8-08dcp-22 & & \\
\hline 1 & 2 & SB8-19dcp-22 & & \\
\hline 1 & 2 & SB8-18dcp-22 & & \\
\hline 1 & 2 & SB8-03dcp-22 & & \\
\hline 1 & 2 & SB8-05dcp-12 & & \\
\hline 1 & 2 & SB8-21dcp-22 & & \\
\hline 1 & 2 & SB8-09dcp-12 & & \\
\hline 1 & 2 & Std-dcp-123 & & \\
\hline 2 & 1 & Std-dcp-211 & & \\
\hline 2 & 1 & SB8-01dcp-21 & & \\
\hline 2 & 1 & SB8-16dcp-11 & & \\
\hline 2 & 1 & SB8-04dcp-11 & & \\
\hline 2 & 1 & SB8-11dcp-11 & & \\
\hline 2 & 1 & SB8-11dcp-21 & & \\
\hline 2 & 1 & SB8-22dcp-21 & & \\
\hline 2 & 1 & SB8-10dcp-11 & & \\
\hline 2 & 1 & SB8-16dcp-21 & & \\
\hline 2 & 1 & Std-dcp-212 & & \\
\hline 2 & 1 & SB8-17dcp-21 & & \\
\hline 2 & 1 & SB8-10dcp-21 & & \\
\hline 2 & 1 & SB8-04dcp-21 & & \\
\hline 2 & 1 & SB8-01dcp-11 & & \\
\hline 2 & 1 & SB8-22dcp-11 & & \\
\hline 2 & 1 & SB8-17dcp-11 & & \\
\hline 2 & 1 & Std-dcp-213 & & \\
\hline
\end{tabular}


SRNL-STI-2012-00725

Revision 0

\begin{tabular}{|c|c|c|c|c|}
\hline $\begin{array}{c}\text { Block } \\
\text { Number }\end{array}$ & $\begin{array}{c}\text { Measurement } \\
\text { Group }\end{array}$ & Sample ID & B & $\mathbf{L i}$ \\
\hline 2 & 2 & Std-dcp-221 & & \\
\hline 2 & 2 & SB8-11dcp-12 & & \\
\hline 2 & 2 & SB8-17dcp-12 & & \\
\hline 2 & 2 & SB8-04dcp-12 & & \\
\hline 2 & 2 & SB8-10dcp-22 & & \\
\hline 2 & 2 & SB8-16dcp-12 & & \\
\hline 2 & 2 & SB8-01dcp-22 & & \\
\hline 2 & 2 & SB8-01dcp-12 & & \\
\hline 2 & 2 & SB8-10dcp-12 & & \\
\hline 2 & 2 & Std-dcp-222 & & \\
\hline 2 & 2 & SB8-16dcp-22 & & \\
\hline 2 & 2 & SB8-22dcp-22 & & \\
\hline 2 & 2 & SB8-22dcp-12 & & \\
\hline 2 & 2 & SB8-11dcp-22 & & \\
\hline 2 & 2 & SB8-17dcp-22 & & \\
\hline 2 & 2 & SB8-04dcp-22 & & \\
\hline 2 & 2 & Std-dcp-223 & & \\
\hline 3 & 1 & Std-dcp-311 & & \\
\hline 3 & 1 & SB8-12dcp-11 & & \\
\hline 3 & 1 & SB8-15dcp-21 & & \\
\hline 3 & 1 & SB8-12dcp-21 & & \\
\hline 3 & 1 & SB8-13dcp-11 & & \\
\hline 3 & 1 & SB8-02dcp-21 & & \\
\hline 3 & 1 & SB8-13dcp-21 & & \\
\hline 3 & 1 & SB8-02dcp-11 & & \\
\hline 3 & 1 & SB8-20dcp-11 & & \\
\hline 3 & 1 & Std-dcp-312 & & \\
\hline 3 & 1 & SB8-14dcp-21 & & \\
\hline 3 & 1 & SB8-07dcp-21 & & \\
\hline 3 & 1 & SB8-20dcp-21 & & \\
\hline 3 & 1 & SB8-14dcp-11 & & \\
\hline 3 & 1 & SB8-15dcp-11 & & \\
\hline 3 & 1 & SB8-07dcp-11 & & \\
\hline 3 & 1 & Std-dcp-313 & & \\
\hline 3 & 2 & Std-dcp-321 & & \\
\hline 3 & 2 & SB8-20dcp-22 & & \\
\hline 3 & 2 & SB8-12dcp-12 & & \\
\hline 3 & 2 & SB8-20dcp-12 & & \\
\hline 3 & 2 & SB8-12dcp-22 & & \\
\hline 3 & 2 & SB8-15dcp-12 & & \\
\hline 3 & 2 & SB8-07dcp-12 & & \\
\hline 3 & 2 & SB8-14dcp-12 & & \\
\hline 3 & 2 & SB8-07dcp-22 & & \\
\hline 3 & 2 & Std-dcp-322 & & \\
\hline 3 & 2 & SB8-15dcp-22 & & \\
\hline 3 & 2 & SB8-14dcp-22 & & \\
\hline 3 & 2 & SB8-13dcp-22 & & \\
\hline 3 & 2 & SB8-02dcp-22 & & \\
\hline 3 & 2 & SB8-02dcp-12 & & \\
\hline 3 & 2 & SB8-13dcp-12 & & \\
\hline 3 & 2 & Std-dcp-323 & & \\
\hline
\end{tabular}


SRNL-STI-2012-00725

Revision 0

\section{Attachment 2.}

\section{Measurement of PCT Solutions ${ }^{f}$}

The PCT solutions are to be measured by Inductively Coupled Plasma - Atomic Emission Spectroscopy (ICP) or by DCP. A multi-element solution standard (denoted by "std-ij" where i = A, $\mathrm{B}, \mathrm{C}, \ldots$, I represents one of the 9 block letters and $\mathrm{j}=1,2$, and 3 represents the position in the block) is to be added at the beginning, middle, and end of each of the nine ICP or DCP blocks by the VSL laboratory technicians. Table A2.1 presents the PCT measurement plan. As the analyses are conducted by VSL, each sample group or block of Table A2.1 requires a different calibration of the ICP or DCP instrumentation. Each of the solution samples of Table A2.1 is to be analyzed by VSL only once for each of the elements of interest to VSL. However, the following elements are to be provided to SRNL for further evaluation: boron (B), lithium (Li), sodium (Na), and silicon (Si) concentrations. The measurements provided to SRNL are to be reported in parts per million (ppm) after accounting for all dilutions and other adjustments conducted during the measurement process. Table A2.2 provides a template for use by VSL in reporting these results. A value below its detection limit should be indicated in this table by a less than sign ("<”) followed by the detection limit.

\footnotetext{
${ }^{f}$ Copies of this attachment are to be provided to the laboratory technicians at VSL who are to conduct ICP or DCP analyses of the PCT solutions.
} 
Table A2.1 ICP or DCP Calibration Blocks for the Leachate Measurements for the PCTs

\begin{tabular}{|c|c|c|c|c|c|c|c|c|}
\hline \multicolumn{3}{|c|}{ PCT Solutions from Oven Runs 1 and 2} & \multicolumn{3}{|c|}{ PCT Solutions from Oven Runs 3 and 4} & \multicolumn{3}{|c|}{ PCT Solutions from Oven Runs 5 and 6} \\
\hline $\begin{array}{c}\text { Calibration } \\
\text { Block A }\end{array}$ & $\begin{array}{c}\text { Calibration } \\
\text { Block B } \\
\end{array}$ & $\begin{array}{c}\text { Calibration } \\
\text { Block C } \\
\end{array}$ & $\begin{array}{c}\text { Calibration } \\
\text { Block D } \\
\end{array}$ & $\begin{array}{c}\text { Calibration } \\
\text { Block E } \\
\end{array}$ & $\begin{array}{c}\text { Calibration } \\
\text { Block F }\end{array}$ & $\begin{array}{c}\text { Calibration } \\
\text { Block G } \\
\end{array}$ & $\begin{array}{c}\text { Calibration } \\
\text { Block H }\end{array}$ & $\begin{array}{c}\text { Calibration } \\
\text { Block I }\end{array}$ \\
\hline std-A1 & std-B1 & std-C1 & std-D1 & std-E1 & std-F1 & std-G1 & std-H1 & std-I1 \\
\hline SB8-pct-074 & SB8-pct-153 & SB8-pct-060 & SB8-pct-164 & SB8-pct-018 & SB8-pct-151 & SB8-pct-002 & SB8-pct-043 & SB8-pct-009 \\
\hline SB8-pct-116 & SB8-pct-050 & SB8-pct-075 & SB8-pct-011 & SB8-pct-087 & SB8-pct-031 & SB8-pct-028 & SB8-pct-044 & SB8-pct-141 \\
\hline SB8-pct-003 & SB8-pct-042 & SB8-pct-068 & SB8-pct-157 & SB8-pct-080 & SB8-pct-073 & SB8-pct-171 & SB8-pct-137 & SB8-pct-046 \\
\hline SB8-pct-083 & SB8-pct-092 & SB8-pct-135 & SB8-pct-159 & SB8-pct-107 & SB8-pct-054 & SB8-pct-019 & SB8-pct-041 & SB8-pct-049 \\
\hline SB8-pct-053 & SB8-pct-056 & SB8-pct-105 & SB8-pct-070 & SB8-pct-063 & SB8-pct-093 & SB8-pct-096 & SB8-pct-128 & SB8-pct-125 \\
\hline SB8-pct-101 & SB8-pct-124 & SB8-pct-104 & SB8-pct-047 & SB8-pct-133 & SB8-pct-072 & SB8-pct-057 & SB8-pct-134 & SB8-pct-025 \\
\hline SB8-pct-076 & SB8-pct-052 & SB8-pct-051 & SB8-pct-013 & SB8-pct-142 & SB8-pct-024 & SB8-pct-062 & SB8-pct-026 & SB8-pct-154 \\
\hline SB8-pct-091 & SB8-pct-110 & SB8-pct-100 & SB8-pct-017 & SB8-pct-172 & SB8-pct-163 & SB8-pct-089 & SB8-pct-166 & SB8-pct-081 \\
\hline SB8-pct-030 & SB8-pct-001 & SB8-pct-132 & SB8-pct-085 & SB8-pct-040 & SB8-pct-082 & SB8-pct-152 & SB8-pct-038 & SB8-pct-174 \\
\hline SB8-pct-008 & SB8-pct-169 & SB8-pct-126 & SB8-pct-099 & SB8-pct-123 & SB8-pct-029 & SB8-pct-114 & SB8-pct-048 & SB8-pct-015 \\
\hline SB8-pct-118 & SB8-pct-178 & SB8-pct-098 & SB8-pct-095 & SB8-pct-165 & SB8-pct-020 & SB8-pct-035 & SB8-pct-005 & SB8-pct-108 \\
\hline std-A2 & std-B2 & std-C2 & std-D2 & std-E2 & std-F2 & std-G2 & std-H2 & std-I2 \\
\hline SB8-pct-149 & SB8-pct-160 & SB8-pct-086 & SB8-pct-146 & SB8-pct-021 & SB8-pct-138 & SB8-pct-102 & SB8-pct-077 & SB8-pct-177 \\
\hline SB8-pct-150 & SB8-pct-088 & SB8-pct-094 & SB8-pct-121 & SB8-pct-090 & SB8-pct-147 & SB8-pct-176 & SB8-pct-012 & SB8-pct-004 \\
\hline SB8-pct-027 & SB8-pct-129 & SB8-pct-022 & SB8-pct-097 & SB8-pct-117 & SB8-pct-007 & SB8-pct-023 & SB8-pct-130 & SB8-pct-168 \\
\hline SB8-pct-158 & SB8-pct-061 & SB8-pct-065 & SB8-pct-136 & SB8-pct-115 & SB8-pct-112 & SB8-pct-119 & SB8-pct-010 & SB8-pct-055 \\
\hline SB8-pct-032 & SB8-pct-180 & SB8-pct-037 & SB8-pct-071 & SB8-pct-156 & SB8-pct-064 & SB8-pct-106 & SB8-pct-173 & SB8-pct-045 \\
\hline SB8-pct-036 & SB8-pct-139 & SB8-pct-140 & SB8-pct-079 & SB8-pct-039 & SB8-pct-122 & SB8-pct-120 & SB8-pct-067 & SB8-pct-127 \\
\hline SB8-pct-143 & SB8-pct-113 & SB8-pct-167 & SB8-pct-078 & SB8-pct-059 & SB8-pct-155 & SB8-pct-006 & SB8-pct-103 & SB8-pct-014 \\
\hline SB8-pct-179 & SB8-pct-161 & SB8-pct-148 & SB8-pct-058 & SB8-pct-144 & SB8-pct-066 & SB8-pct-084 & SB8-pct-034 & SB8-pct-109 \\
\hline SB8-pct-145 & SB8-pct-069 & SB8-pct-170 & std-D3 & SB8-pct-162 & std-F3 & std-G3 & SB8-pct-016 & std-I3 \\
\hline SB8-pct-175 & SB8-pct-131 & SB8-pct-033 & & std-E3 & & & std-H3 & \\
\hline \multirow[t]{2}{*}{ std-A3 } & SB8-pct-111 & std-C3 & & & & & & \\
\hline & std-B3 & & & & & & & \\
\hline
\end{tabular}


SRNL-STI-2012-00725

Revision 0

Table A2.2 Reporting Template for the Leachate Measurements for the PCTs

\begin{tabular}{|c|c|c|c|c|}
\hline \multicolumn{5}{|l|}{ PCT Solutions from Oven Runs 1 and 2} \\
\hline Calibration Block A & B - boron (ppm) & Li - lithium (ppm) & $\mathrm{Na}$ - sodium (ppm) & Si - Silicon (ppm) \\
\hline std-A1 & & & & \\
\hline SB8-pct-074 & & & & \\
\hline SB8-pct-116 & & & & \\
\hline SB8-pct-003 & & & & \\
\hline SB8-pct-083 & & & & \\
\hline SB8-pct-053 & & & & \\
\hline SB8-pct-101 & & & & \\
\hline SB8-pct-076 & & & & \\
\hline SB8-pct-091 & & & & \\
\hline SB8-pct-030 & & & & \\
\hline SB8-pct-008 & & & & \\
\hline SB8-pct-118 & & & & \\
\hline std-A2 & & & & \\
\hline SB8-pct-149 & & & & \\
\hline SB8-pct-150 & & & & \\
\hline SB8-pct-027 & & & & \\
\hline SB8-pct-158 & & & & \\
\hline SB8-pct-032 & & & & \\
\hline SB8-pct-036 & & & & \\
\hline SB8-pct-143 & & & & \\
\hline SB8-pct-179 & & & & \\
\hline SB8-pct-145 & & & & \\
\hline SB8-pct-175 & & & & \\
\hline std-A3 & & & & \\
\hline Calibration Block B & B - boron (ppm) & Li - lithium (ppm) & $\mathrm{Na}$ - sodium (ppm) & Si - Silicon (ppm) \\
\hline std-B1 & & & & \\
\hline SB8-pct-153 & & & & \\
\hline SB8-pct-050 & & & & \\
\hline SB8-pct-042 & & & & \\
\hline SB8-pct-092 & & & & \\
\hline SB8-pct-056 & & & & \\
\hline SB8-pct-124 & & & & \\
\hline SB8-pct-052 & & & & \\
\hline SB8-pct-110 & & & & \\
\hline SB8-pct-001 & & & & \\
\hline SB8-pct-169 & & & & \\
\hline SB8-pct-178 & & & & \\
\hline std-B2 & & & & \\
\hline SB8-pct-160 & & & & \\
\hline SB8-pct-088 & & & & \\
\hline SB8-pct-129 & & & & \\
\hline SB8-pct-061 & & & & \\
\hline SB8-pct-180 & & & & \\
\hline SB8-pct-139 & & & & \\
\hline SB8-pct-113 & & & & \\
\hline SB8-pct-161 & & & & \\
\hline SB8-pct-069 & & & & \\
\hline SB8-pct-131 & & & & \\
\hline SB8-pct-111 & & & & \\
\hline std-B3 & & & & \\
\hline & & & & \\
\hline
\end{tabular}


SRNL-STI-2012-00725

Revision 0

\begin{tabular}{|c|c|c|c|c|}
\hline Calibration Block C & B - boron (ppm) & Li - lithium (ppm) & $\mathrm{Na}$ - sodium (ppm) & Si - Silicon (ppm) \\
\hline std-C1 & & & & \\
\hline SB8-pct-060 & & & & \\
\hline SB8-pct-075 & & & & \\
\hline SB8-pct-068 & & & & \\
\hline SB8-pct-135 & & & & \\
\hline SB8-pct-105 & & & & \\
\hline SB8-pct-104 & & & & \\
\hline SB8-pct-051 & & & & \\
\hline SB8-pct-100 & & & & \\
\hline SB8-pct-132 & & & & \\
\hline SB8-pct-126 & & & & \\
\hline SB8-pct-098 & & & & \\
\hline std-C2 & & & & \\
\hline SB8-pct-086 & & & & \\
\hline SB8-pct-094 & & & & \\
\hline SB8-pct-022 & & & & \\
\hline SB8-pct-065 & & & & \\
\hline SB8-pct-037 & & & & \\
\hline SB8-pct-140 & & & & \\
\hline SB8-pct-167 & & & & \\
\hline SB8-pct-148 & & & & \\
\hline SB8-pct-170 & & & & \\
\hline SB8-pct-033 & & & & \\
\hline std-C3 & & & & \\
\hline PCT Solutions from Oven Runs 3 and 4 & & & & \\
\hline Calibration Block D & B - boron (ppm) & Li - lithium (ppm) & $\mathrm{Na}$ - sodium (ppm) & Si - Silicon (ppm) \\
\hline std-D1 & & & & \\
\hline SB8-pct-164 & & & & \\
\hline SB8-pct-011 & & & & \\
\hline SB8-pct-157 & & & & \\
\hline SB8-pct-159 & & & & \\
\hline SB8-pct-070 & & & & \\
\hline SB8-pct-047 & & & & \\
\hline SB8-pct-013 & & & & \\
\hline SB8-pct-017 & & & & \\
\hline SB8-pct-085 & & & & \\
\hline SB8-pct-099 & & & & \\
\hline SB8-pct-095 & & & & \\
\hline std-D2 & & & & \\
\hline SB8-pct-146 & & & & \\
\hline SB8-pct-121 & & & & \\
\hline SB8-pct-097 & & & & \\
\hline SB8-pct-136 & & & & \\
\hline SB8-pct-071 & & & & \\
\hline SB8-pct-079 & & & & \\
\hline SB8-pct-078 & & & & \\
\hline SB8-pct-058 & & & & \\
\hline std-D3 & & & & \\
\hline & & & & \\
\hline
\end{tabular}


SRNL-STI-2012-00725

Revision 0

\begin{tabular}{|c|c|c|c|c|}
\hline Calibration Block E & B - boron (ppm) & Li - lithium (ppm) & $\mathrm{Na}$ - sodium (ppm) & Si - Silicon (ppm) \\
\hline \multicolumn{5}{|l|}{ std-E1 } \\
\hline \multicolumn{5}{|l|}{ SB8-pct-018 } \\
\hline \multicolumn{5}{|l|}{ SB8-pct-087 } \\
\hline \multirow{2}{*}{\multicolumn{5}{|c|}{ SB8-pct-080 }} \\
\hline \multirow{2}{*}{\multicolumn{5}{|c|}{$\begin{array}{l}\text { SB8-pct-107 } \\
\text { SB8-pct-063 }\end{array}$}} \\
\hline & & & & \\
\hline \multicolumn{5}{|l|}{ SB8-pct-133 } \\
\hline \multicolumn{5}{|l|}{ SB8-pct-142 } \\
\hline \multicolumn{5}{|l|}{ SB8-pct-172 } \\
\hline \multicolumn{5}{|l|}{ SB8-pct-040 } \\
\hline \multicolumn{5}{|l|}{ SB8-pct-123 } \\
\hline \multicolumn{5}{|l|}{ SB8-pct-165 } \\
\hline \multicolumn{5}{|l|}{ std-E2 } \\
\hline \multicolumn{5}{|l|}{ SB8-pct-021 } \\
\hline \multirow{2}{*}{\multicolumn{5}{|c|}{ SB8-pct-090 }} \\
\hline \multicolumn{2}{|l|}{$\begin{array}{l}\text { SB8-pct-117 } \\
\text { SB8-nt } 115\end{array}$} & & & \\
\hline \multirow{2}{*}{\multicolumn{5}{|c|}{$\begin{array}{l}\text { SB8-pct-115 } \\
\text { SB8-pct-156 }\end{array}$}} \\
\hline & & & & \\
\hline \multicolumn{5}{|l|}{ SB8-pct-039 } \\
\hline \multicolumn{5}{|l|}{ SB8-pct-059 } \\
\hline \multicolumn{5}{|l|}{ SB8-pct-144 } \\
\hline \multicolumn{5}{|l|}{$\frac{\text { SB8-pct-162 }}{\text { std-E3 }}$} \\
\hline \multicolumn{5}{|l|}{ std-E3 } \\
\hline Calibration Block F & B - boron (ppm) & Li - lithium (ppm) & $\mathrm{Na}$ - sodium (ppm) & Si - Silicon (ppm) \\
\hline \multicolumn{5}{|l|}{ std-F1 } \\
\hline \multicolumn{5}{|l|}{ SB8-pct-151 } \\
\hline SB8-pct-031 & & & & \\
\hline SB8-pct-073 & & & & \\
\hline SB8-pct-054 & & & & \\
\hline SB8-pct-093 & & & & \\
\hline SB8-pct-072 & & & & \\
\hline SB8-pct-024 & & & & \\
\hline SB8-pct-163 & & & & \\
\hline SB8-pct-082 & & & & \\
\hline SB8-pct-029 & & & & \\
\hline SB8-pct-020 & & & & \\
\hline std-F2 & & & & \\
\hline SB8-pct-138 & & & & \\
\hline SB8-pct-147 & & & & \\
\hline SB8-pct-007 & & & & \\
\hline SB8-pct-112 & & & & \\
\hline SB8-pct-064 & & & & \\
\hline SB8-pct-122 & & & & \\
\hline SB8-pct-155 & & & & \\
\hline SB8-pct-066 & & & & \\
\hline std-F3 & & & & \\
\hline
\end{tabular}


SRNL-STI-2012-00725

Revision 0

\begin{tabular}{|c|c|c|c|c|}
\hline \multicolumn{5}{|l|}{ PCT Solutions from Oven Runs 5 and 6} \\
\hline Calibration Block G & B - boron (ppm) & Li - lithium (ppm) & Na - sodium (ppm) & Si - Silicon (ppm) \\
\hline std-G1 & & & & \\
\hline SB8-pct-002 & & & & \\
\hline SB8-pct-028 & & & & \\
\hline SB8-pct-171 & & & & \\
\hline SB8-pct-019 & & & & \\
\hline SB8-pct-096 & & & & \\
\hline SB8-pct-057 & & & & \\
\hline SB8-pct-062 & & & & \\
\hline SB8-pct-089 & & & & \\
\hline SB8-pct-152 & & & & \\
\hline SB8-pct-114 & & & & \\
\hline SB8-pct-035 & & & & \\
\hline std-G2 & & & & \\
\hline SB8-pct-102 & & & & \\
\hline SB8-pct-176 & & & & \\
\hline SB8-pct-023 & & & & \\
\hline SB8-pct-119 & & & & \\
\hline SB8-pct-106 & & & & \\
\hline SB8-pct-120 & & & & \\
\hline SB8-pct-006 & & & & \\
\hline SB8-pct-084 & & & & \\
\hline std-G3 & & & & \\
\hline Calibration Block H & B - boron (ppm) & Li - lithium (ppm) & Na - sodium (ppm) & Si - Silicon (ppm) \\
\hline std-H1 & & & & \\
\hline SB8-pct-043 & & & & \\
\hline SB8-pct-044 & & & & \\
\hline SB8-pct-137 & & & & \\
\hline SB8-pct-041 & & & & \\
\hline SB8-pct-128 & & & & \\
\hline SB8-pct-134 & & & & \\
\hline SB8-pct-026 & & & & \\
\hline SB8-pct-166 & & & & \\
\hline SB8-pct-038 & & & & \\
\hline SB8-pct-048 & & & & \\
\hline SB8-pct-005 & & & & \\
\hline std-H2 & & & & \\
\hline SB8-pct-077 & & & & \\
\hline SB8-pct-012 & & & & \\
\hline SB8-pct-130 & & & & \\
\hline SB8-pct-010 & & & & \\
\hline SB8-pct-173 & & & & \\
\hline SB8-pct-067 & & & & \\
\hline SB8-pct-103 & & & & \\
\hline SB8-pct-034 & & & & \\
\hline SB8-pct-016 & & & & \\
\hline std-H3 & & & & \\
\hline
\end{tabular}


SRNL-STI-2012-00725

Revision 0

\begin{tabular}{|c|l|l|l|l|}
\hline Calibration Block I & B - boron (ppm) & Li - lithium (ppm) & Na - sodium (ppm) & Si - Silicon (ppm) \\
\hline std-I1 & & & & \\
\hline SB8-pct-009 & & & & \\
\hline SB8-pct-141 & & & & \\
\hline SB8-pct-046 & & & & \\
\hline SB8-pct-049 & & & & \\
\hline SB8-pct-125 & & & & \\
\hline SB8-pct-025 & & & & \\
\hline SB8-pct-154 & & & & \\
\hline SB8-pct-081 & & & & \\
\hline SB8-pct-174 & & & & \\
\hline SB8-pct-015 & & & & \\
\hline SB8-pct-108 & & & & \\
\hline std-I2 & & & & \\
\hline SB8-pct-177 & & & & \\
\hline SB8-pct-004 & & & & \\
\hline SB8-pct-168 & & & & \\
\hline SB8-pct-055 & & & & \\
\hline SB8-pct-045 & & & & \\
\hline SB8-pct-127 & & & & \\
\hline SB8-pct-014 & & & & \\
\hline SB8-pct-109 & & & & \\
\hline std-I3 & & & & \\
\hline
\end{tabular}




\section{Electronic Distribution by E-Mail to the Following}

\section{SRS Distribution:}

\begin{tabular}{|l|l|}
\hline Name: & Location: \\
\hline Patrick R. Jackson & $703-46 \mathrm{~A}$ \\
\hline Sharon Marra & $773-\mathrm{A}$ \\
\hline Connie Herman & $999-\mathrm{W}$ \\
\hline Patricia Lee & $703-41 \mathrm{~A}$ \\
\hline Rachel Baker & $703-41 \mathrm{~A}$ \\
\hline David Peeler & $999-\mathrm{W}$ \\
\hline Tommy Edwards & $999-\mathrm{W}$ \\
\hline \hline Kevin Fox & $999-\mathrm{W}$ \\
\hline \hline Eric Freed & $704-\mathrm{S}$ \\
\hline \hline Dave Sherburne & $704-\mathrm{S}$ \\
\hline \hline Kishor Shah & $704-\mathrm{S}$ \\
\hline \hline Karthik Subramanian & $766-\mathrm{H}$ \\
\hline \hline Jonathan Bricker & $704-27 \mathrm{~S}$ \\
\hline \hline John Iaukea & $704-30 \mathrm{~S}$ \\
\hline \hline Jeff Ray & $704-\mathrm{S}$ \\
\hline \hline Robert Hinds & $704-\mathrm{S}$ \\
\hline Terri Fellinger & $704-26 \mathrm{~S}$ \\
\hline \hline Amanda Shafer & $704-27 \mathrm{~S}$ \\
\hline \hline Mason Clark & $704-27 \mathrm{~S}$ \\
\hline \hline Helen P. Boyd & $704-15 \mathrm{~S}$ \\
\hline Hank Elder & \\
\hline Bill Holtzscheiter & \\
\hline Pat Vaughan & \\
\hline
\end{tabular}

\section{CUA/VSL Distribution:}

\begin{tabular}{|l|l|}
\hline Name: & Location: \\
\hline Wing Kot & CUA/VSL \\
\hline Ian Pegg & CUA/VSL \\
\hline & \\
\hline & \\
\hline & \\
\hline & \\
\hline & \\
\hline
\end{tabular}

\section{ES Distribution:}

\begin{tabular}{|l|l|}
\hline Name: & Location: \\
\hline Brad Bowan & ES \\
\hline Glenn Diener & ES \\
\hline Innocent Joseph & ES \\
\hline & \\
\hline & \\
\hline & \\
\hline & \\
\hline
\end{tabular}

